\title{
$N$-Glycosylation of Acid-Sensing Ion Channel la Regulates Its Trafficking and Acidosis-Induced Spine Remodeling
}

\author{
Lan Jing, ${ }^{1,2 *}$ Xiang-Ping Chu, ${ }^{3 \star}$ Yu-Qing Jiang, ${ }^{1,4 \star}$ Daniel M. Collier, ${ }^{5}$ Bin Wang, ${ }^{6}$ Qian Jiang, ${ }^{3}$ Peter M. Snyder, ${ }^{5}$ \\ and Xiang-Ming Zha ${ }^{1}$ \\ ${ }^{1}$ Department of Cell Biology and Neuroscience, University of South Alabama College of Medicine, Mobile, Alabama 36688, ${ }^{2}$ State Key Laboratory of New \\ Drug \& Pharmaceutical Process, Shanghai Institute of Pharmaceutical Industry, Shanghai 200040, China, ${ }^{3}$ Departments of Basic Medical Science and \\ Anesthesiology, University of Missouri-Kansas City School of Medicine, Kansas City, Missouri 64108, ${ }^{4}$ Department of Urology, Third Hospital of Hebei \\ Medical University, Shijiazhuang, Hebei 050051, China, ${ }^{5}$ Departments of Internal Medicine and Molecular Physiology and Biophysics, University of Iowa, \\ Iowa City, Iowa 52242, and 'Department of Mathematics and Statistics, University of South Alabama, Mobile, Alabama 36688
}

Acid-sensing ion channel-1a (ASIC1a) is a potential therapeutic target for multiple neurological diseases. We studied here ASIC1a glycosylation and trafficking, two poorly understood processes pivotal in determining the functional outcome of an ion channel. We found that most ASIC1a in the mouse brain was fully glycosylated. Inhibiting glycosylation with tunicamycin reduced ASIC1a surface trafficking, dendritic targeting, and acid-activated current density. $N$-glycosylation of the two glycosylation sites, Asn393 and Asn366, has differential effects on ASIC1a biogenesis. Maturation of Asn393 increased ASIC1a surface and dendritic trafficking, pH sensitivity, and current density. In contrast, glycosylation of Asn366 was dispensable for ASIC1a function and may be a rate-limiting step in ASIC1a biogenesis. In addition, we revealed that acidosis reduced the density and length of dendritic spines in a time- and ASIC1a-dependent manner. ASIC1a N366Q, which showed increased glycosylation and dendritic targeting, potentiated acidosis-induced spine loss. Conversely, ASIC1a N393Q, which had diminished dendritic targeting and inhibited ASIC1a current dominant-negatively, had the opposite effect. These data tie $\mathrm{N}$-glycosylation of ASIC1a with its trafficking. More importantly, by revealing a site-specific effect of acidosis on dendritic spines, our findings suggest that these processes have an important role in regulating synaptic plasticity and determining long-term consequences in diseases that generate acidosis.

\section{Introduction}

Brain acidosis, or a reduction in extracellular brain $\mathrm{pH}$, leads to neuronal damage in multiple neurological diseases (Siesjö, 1982; Sluka et al., 2009). However, little is known about whether acidosis regulates synaptic remodeling, which has a critical impact on neuron physiology (Bhatt et al., 2009). Answering this question will expand our current focus on the toxic effects of acidosis on neuronal cell bodies to include synaptic sites, and advance our understanding of the long-term changes in neuroplasticity in diseases that generate acidosis.

Received Oct. 3, 2011; revised Jan. 10, 2012; accepted Jan. 31, 2012.

Author contributions: L.J., X.-P.C., Y.-Q.J., P.M.S., and X.-M.Z. designed research; L.J., X.-P.C., Y.-Q.J., D.M.C., Q.J., and X.-M.Z. performed research; L.J., X.-P.C., D.M.C., B.W., P.M.S., and X.-M.Z. analyzed data; L.J., X.-P.C., P.M.S., and X.-M.Z. wrote the paper.

This work was supported by grants from the National Institutes of Health (R21 Grant \#DA031259), the American Heart Association (Grant \#0735092N), and the University of Missouri Research Board (X.-P.C.); a fellowship grant from The Third Hospital of Hebei Medical University (Y.-Q.J.), National Institutes of Health Grant HL072256 (P.M.S.), and startup funds from University of South Alabama (X.-M.Z.). The Nikon A1 confocal microscope was funded by National Institutes of Health/American Recovery \& Reinvestment Act Equipment Grant \#S10RR027535. We thank Drs. Michael Welsh and John Wemmie for providing the $A S I C 1 a^{-/-}$mice and the rabbit anti-ASIC1 antibody, Dr. Michel Lazdunski for the rat ASIC1a construct, and Dr. Steven Green for the Lck-GFP construct.

*L.J., X.P.C. and Y.-Q.J. contributed equally to this work.

Correspondence should be addressed to Xiang-Ming Zha, Department of Cell Biology and Neuroscience, University of South Alabama College of Medicine, 307 University Boulevard, MSB1201, Mobile, AL 36688. E-mail: zha@jaguar1.usouthal.edu.

DOI:10.1523/JNEUROSCI.5021-11.2012

Copyright $\odot 2012$ the authors $\quad 0270-6474 / 12 / 324080-12 \$ 15.00 / 0$
The key mediator of extracellular acidosis is acid-sensing ion channel-1a (ASIC1a), the major proton receptor in the brain (Waldmann et al., 1997; Wemmie et al., 2002; Noël et al., 2010). ASIC1a mediates acidosis-induced neuronal injury in ischemia and multiple sclerosis (Xiong et al., 2004; Yermolaieva et al., 2004; Gao et al., 2005; Friese et al., 2007). ASIC1a also regulates neuronal firing and synaptic plasticity, and contributes to seizure termination (Wemmie et al., 2002; Vukicevic and Kellenberger, 2004; Cho and Askwith, 2008; Ziemann et al., 2008). At the cellular level, ASIC1a localizes to dendrites and dendritic spines, the sites for excitatory neurotransmission in the brain, and mediates acid-induced intracellular $\mathrm{Ca}^{2+}$ concentration $\left(\left[\mathrm{Ca}^{2+}\right]_{\mathrm{i}}\right)$ change in dendritic region (Zha et al., 2006; Bhatt et al., 2009). Together, these data emphasize the importance of ASIC1a in acidosisinduced neuronal injury and spine remodeling. Thus, a better understanding of the basic biological processes regulating ASIC1a will provide important insight into the etiology of multiple diseases.

One fundamental way to regulate an ion channel is by modulating its trafficking, which is tightly controlled by its maturation through the secretory pathway (Hebert et al., 2005). However, there is a dearth of knowledge concerning ASICla maturation and its role in ASICla trafficking and function. In one study, mutating the glycosylation sites of ASICla decreased its current amplitude in Xenopus oocytes (Kadurin et al., 2008). Also, as we showed recently, extracellular mutants with reduced glycosyla- 
tion exhibit reduced surface trafficking in Chinese hamster ovary (CHO) cells (Jing et al., 2011). While these results are interesting, no experiments have directly assessed whether $\mathrm{N}$-glycosylation regulates ASIC1a surface expression. Besides surface expression, trafficking in neurons contains another important aspect-the targeting to distinct neuronal compartments, including dendrites, axons, and synaptic sites. Although it is known that ASIC1a preferentially localizes to dendrites (Zha et al., 2006, 2009b), it remains unclear whether the maturation process regulates ASIC1a trafficking to the dendritic region.

Here, we investigated the role of glycosylation in ASICla trafficking and acidosis-induced dendritic remodeling. We found that the majority of ASICla in the brain is fully glycosylated, and maturation of $\mathrm{N}$-glycans is necessary for efficient surface expression and dendritic targeting of ASIC1a. ASIC1a glycosylation further regulates ASICla pH sensitivity and acid-activated current density. These findings are the first to reveal the importance of $\mathrm{N}$-glycosylation in ASICla trafficking and function in brain neurons. More importantly, our data on acid-induced spine remodeling suggest a novel direction for future investigation of how acidosis and ASICs regulate plasticity in diseases.

\section{Materials and Methods}

Mice. ASIC1 $a^{-1-}$ mice on a congenic C57BL/6 background were kindly provided by Drs. Michael Welsh and John Wemmie (Howard Hughes Medical Institute and University of Iowa, Iowa City, IA). Wild-type and knock-out mice were maintained as described previously (Zha et al., 2009b). Postnatal day 6 (P6) pups of either sex or P15-P16 males were used for this study. Animal care met National Institutes of Health standards and all procedures were approved by the University of South Alabama Animal Care and Use Committee.

Constructs and reagents. Lck-GFP and rat ASICla were kindly provided by Drs. Steven Green (University of Iowa, Iowa CIty, IA) and Michel Lazdunski (Institut de Pharmacologie Moléculaire et Cellulaire, Valbonne, France), respectively. Constructs encoding mouse ASIC1a and $\mathrm{N}$-terminal hemagglutinin (HA)-tagged mouse ASIC1a have been described previously (Zha et al., 2006, 2009a,b; Jing et al., 2011). HA-tag was added to human ASICla by PCR-mediated subcloning. Mouse N366Q, N366A, N393Q, and N366Q/N393Q (QQ) mutants, the rat N366Q mutant, and the human N368Q mutant were generated with a QuikChange mutagenesis kit (Agilent Technologies), following the manufacturer's instructions. All constructs were verified by sequencing. Rabbit anti-ASIC1 was kindly provided by Dr. John Wemmie (Wemmie et al., 2003). Other antibodies used were as follows: rat monoclonal anti-HA (Roche), mouse anti-tubulin (Hybridoma Bank), mouse anti-HA (Syd Labs), goat anti-ASIC1 (Santa Cruz Biotechnology), and Alexa 568-, 680-, 800-, and Dylight 680-conjugated secondary antibodies (Invitrogen, LI-COR Biosciences, and Pierce). Other reagents used were as follows: endoglycosidase $\mathrm{H}$ (endo $\mathrm{H}$ ) and peptide $\mathrm{N}$-glycosidase F (PNGase F) (New England Biolabs); NHS-sulfo-LC-biotin and NeutrAvidin Beads (Pierce); tunicamycin (Thermo Fisher Scientific); culture media and serum (HyClone and Invitrogen); Fugene HD (Roche); and Lipofectamine 2000 (Invitrogen).

$\mathrm{CHO}$ cell culture and transfection. $\mathrm{CHO}-\mathrm{K} 1$ cells were purchased from ATCC and used between passages 3 and 14. CHO cells were grown in F-12K supplemented with $10 \%$ fetal bovine serum in a humidified $5 \%$ $\mathrm{CO}_{2}$ incubator. For biochemistry experiments, cells were plated onto 60 $\mathrm{mm}$ dishes at a density of $3-4 \times 10^{4}$ cells $/ \mathrm{cm}^{2}\left(6-8 \times 10^{5}\right.$ per $60 \mathrm{~mm}$ dish) and transfected using Lipofectamine 2000, following the manufacturer's instruction. For electrophysiology, cells were grown in $35 \mathrm{~mm}$ dishes at a density of $50-70 \%$ and transfected using Fugene HD following the manufacturer's instruction. For tunicamycin treatment, cells were treated with $0.5 \mu \mathrm{g} / \mathrm{ml}$ tunicamycin for $24 \mathrm{~h}$ before analysis.

Deglycosylation. Cell lysate was deglycosylated with PNGase F or endo $\mathrm{H}$ similar to what was described previously (Jing et al., 2011). Briefly, the samples were denatured at $95^{\circ} \mathrm{C}$ for $10 \mathrm{~min}$ and cooled to room temperature. For PNGase F digestion, NP-40 was added to a final concentration of $1 \%$. The reaction mixture was incubated overnight at $37^{\circ} \mathrm{C}$ with PNGase F [ $\geq 0.08$ IUB milliunit (mIUB) for CHO lysate or $\geq 0.6 \mathrm{mIUB}$ for brain lysate] or endo $\mathrm{H}$ ( $\geq 1 \mathrm{mIUB}$ for $\mathrm{CHO}$ lysate or $\geq 4 \mathrm{mIUB}$ for brain lysate) per $300 \mu \mathrm{g}$ of total proteins. The amount of enzymes used ensured a complete deglycosylation based on our titration experiments. Following PNGase F or endo $\mathrm{H}$ treatment, isolation and analysis of surface proteins were performed as described below.

Surface biotinylation, NeutrAvidin pull-down, and Western blot. Surface biotinylation and NeutrAvidin pull-down were performed similar to what has been described previously (Zha et al., 2009a; Jing et al., 2011). For biotinylation of organotypic hippocampal slices, each filter, which contained 6-8 slices, was cut out and put in a six-well plate. $\mathrm{CHO}$ cells or slices were then washed three times with ice-cold PBS $+/+$, followed by 30 min incubation at $4^{\circ} \mathrm{C}$ in $1.5 \mathrm{ml}$ (for $\mathrm{CHO}$ cells in $60 \mathrm{~mm}$ dishes) or 1 $\mathrm{ml}$ (for slices in each well of the 6-well plate) of PBS $+/+$ containing 0.5 $\mathrm{mg} / \mathrm{ml}$ Sulfo-NHS-LC-Biotin. Cells were washed once with cold $\mathrm{PBS}+/+$ and the reaction was quenched by $100 \mathrm{~mm}$ glycine in $\mathrm{PBS}+/+$. Of note, it is essential, especially for biotinylation of slices, to keep the solution and plates ice cold during the whole procedure. Cells were lysed in $300 \mu \mathrm{l}$ of NeutrAvidin lysis buffer (PBS, 1\% Triton, $0.5 \%$ SDS, 0.5 $\mathrm{mg} / \mathrm{ml} \mathrm{N}$-ethylmaleimide, with protease inhibiters). Cell lysates were sonicated briefly and centrifuged at full speed with a desktop centrifuge for $10 \mathrm{~min}$ at $4^{\circ} \mathrm{C}$. For precipitation of surface proteins, $40 \mu \mathrm{l}$ of NeutrAvidin agarose beads were added to $200 \mu \mathrm{l}$ of cell lysate $(\sim 400-600 \mu \mathrm{g}$ of proteins) and the precipitation was performed overnight at $4{ }^{\circ} \mathrm{C}$ with gentle rotation, followed by three washes with PBS containing $1 \%$ Triton.

Surface fraction was eluted with $80 \mu \mathrm{l}$ of $2 \times$ sample buffer containing $5 \% \beta$-mercaptoethanol. Total lysate was mixed with an equal volume of sample buffer. Equal volume of surface and total fraction was loaded per lane so the loading into the surface fraction was $\sim 5 \times$ that of total. The samples were separated by $8 \%$ or $10 \%$ SDS-PAGE and transferred to nitrocellulose membranes. We eliminated SDS in our gel, which appeared to give better separation of the proteins (SDS was still present in both the sample buffer and the running buffer). Membranes were blocked in blocking buffer $(0.1 \%$ casein in $0.2 \times \mathrm{PBS}, \mathrm{pH} 7.4)$ for $1 \mathrm{~h}$. Primary antibodies were diluted with blocking buffer containing $0.1 \%$ Tween 20 and incubated at $4^{\circ} \mathrm{C}$ overnight or at room temperature for $2 \mathrm{~h}$. Secondary antibodies were diluted in blocking buffer containing $0.1 \%$ Tween 20 and $0.01 \%$ SDS and incubated at room temperature for $1 \mathrm{~h}$. Antibody dilutions were as follows: rabbit anti-ASIC1a, 1:8000-30,000; goat anti-ASIC1, 1:500; monoclonal anti-HA, 1:1000-2000; monoclonal anti-tubulin, 1:30,000-60,000; Alexa 680-, 800-, or Dylight $800-$ conjugated secondary antibodies, 1:12,000-16,000. Blots were imaged using an Odyssey Infrared Imaging System according to manufacturer's instructions. Densitometry of imaged bands was performed using NIH ImageJ as described previously (Zha et al., 2006, 2009b).

Hippocampal slice culture, transfection, and immunofluorescence. Organotypic mouse hippocampal slice culture was performed similar to the procedures described previously (Zha et al., 2005, 2006; Jing et al., 2011). Briefly, hippocampi from postnatal day 5-7 pups were cut into $350 \mu \mathrm{m}$ thick sections and cultured in Falcon polyethylene terephthalate-etched membrane culture inserts containing $1 \mu \mathrm{m}$ pores (Thermo Fisher Scientific), at a density of 5-6 slices per insert (for biotinylation experiments, slices were cultured at $6-8$ slices per insert). Slices were maintained in filter culture medium containing the following: $25 \%$ horse serum, $25 \%$ Hanks Balanced Salt Solution, and 50\% MEM, supplemented with $2 \mathrm{mM}$ glutamax, $1.5 \mathrm{mg} / \mathrm{ml}$ glucose, $44 \mathrm{mg} / \mathrm{ml} \mathrm{NaHCO}_{3}$, and $10 \mathrm{U} / \mathrm{ml}$ penicillin-streptomycin. Slices were grown in a $5 \% \mathrm{CO}_{2}$ humidified incubator. The medium was changed every $2-3 \mathrm{~d}$.

Transfection was performed after $8-10 \mathrm{~d}$ in culture with a Helios genegun (Bio-Rad) at 70-75 psi, similar to what has been described previously (Zha et al., 2006, 2009b). For spine analysis, all slices were fixed $2 \mathrm{~d}$ after transfection, at an age equivalent of postnatal day 17 (e.g., P6 + 11 DIV), except that one set of the ASIC1a knock-out slices were fixed at P5 +10 DIV. When needed, slices were treated with medium of different $\mathrm{pH}$ for 30,60 , and $90 \mathrm{~min}$ before fixation. Medium $\mathrm{pH}$ was buffered with $10 \mathrm{~mm}$ HEPES and $10 \mathrm{~mm}$ MES or with $20 \mathrm{~mm}$ MES. The osmolarity of medium at different pHs was adjusted to be $308-318$ mOsm, within the same range of filter culture medium. To study the 
effect of tunicamycin on dendritic targeting, slices were treated with $0.5 \mu \mathrm{g} / \mathrm{ml}$ tunicamycin for $24 \mathrm{~h}$ before fixation. One technical note is that while the general dendritic arborization appeared intact after tunicamycin treatment, many neurons showed blebbed or uneven dendrites. Although this caveat did not affect our quantification of dendritic ASIC1a levels at low magnification, high-resolution imaging of spines or quantification of spine ASIC1a levels is not practical after tunicamycin treatment. Slices were fixed with $4 \%$ paraformaldehyde in Hanks' balanced salt solution $+/+$ containing $6 \mathrm{mg} / \mathrm{ml}$ glucose and $20 \mathrm{~mm}$ HEPES, pH 7.3, for 10-15 min, followed by three washes with $\mathrm{PBS}+/+$. Immunofluorescence was performed as described previously (Zha et al., 2006).

Confocal microscopy and analysis. Confocal images were captured using a laser scanning microscope (Nikon A1), similar to what has been described previously (Jing et al., 2011). Briefly, illumination was provided by an argon (Ar, 458, 488, $514 \mathrm{~nm}$ lines) and a $561 \mathrm{~nm}$ diode laser. To eliminate bleedthrough, green and red channels were imaged sequentially using $488 \mathrm{~nm}$ excitation and a 525/50 emission filter and $561 \mathrm{~nm}$ excitation and a 595/50 emission filter, respectively. Images were captured with a $20 \times / 0.75$ multi-immersion lens or a $63 \times / 1.2 \mathrm{PL}$ APO water lens. To visualize spines, a series of high-resolution images $(1024 \times 256$ to $1024 \times 1024$ pixel array) were captured at a $z$-step of $0.4-0.5 \mu \mathrm{m}$ with an additional electronic zoom of 4 , with an average of four scans in each single plane. For each transfected neuron, one middle segment of an apical dendrite ( $\sim 100-200 \mu \mathrm{m}$ away from the cell body layer) was imaged and used for spine analysis. The image field of view was $\sim 50-70 \mu \mathrm{m}$ and thus covered a large fraction of the medial portion of an apical dendrite. Raw images were exported and further analyzed in NIH ImageJ.

All spine quantifications were done with the observer blinded to experimental conditions. All images were analyzed using the 3D stacks, similar to what has been described previously (Zha et al., 2005, 2006). For quantification of ASICla immunofluorescence in spines, a line was drawn across the spines and their adjacent shafts, and raw ASIC immunofluorescence and GFP fluorescence intensities were measured. The maximum value was used for a given spine or shaft area. As a control, spine/shaft ratio of ASIC was normalized to that of Lck-GFP. Similarly, a line profile analysis was performed to quantify the relative ASIC1a levels in the apical dendrite and cell body.

Electrophysiology in $\mathrm{CHO}$ cells. Whole-cell patch-clamp recordings in CHO cells were performed as described previously (Jiang et al., 2009, 2010, 2011). Briefly, patch electrodes, constructed from thin-walled borosilicated glass, have resistance ranging from 3 to $6 \mathrm{M} \Omega$ when filled with intracellular solution (mM) as follows: $140 \mathrm{~K}$-gluconate, $10 \mathrm{HEPES}$, 11 EGTA, 2 TEA, $1 \mathrm{CaCl}_{2}, 2 \mathrm{MgCl}_{2}$, and $4 \mathrm{~K}_{2} \mathrm{ATP}$, pH 7.2-7.3 (290-300 $\mathrm{mOsm})$. Whole-cell currents were elicited by a drop in $\mathrm{pH}$ from 7.4 to different $\mathrm{pHs}$ at a holding potential of $-60 \mathrm{mV}$ and recorded using Axopatch 200B amplifiers (Axon CNS, Molecular Devices). Data were filtered at $2 \mathrm{kHz}$ and digitized at $5 \mathrm{~Hz}$, and acquired using pClamp. Standard extracellular fluid (ECF) contained the following (in mM): 140 $\mathrm{NaCl}, 5.4 \mathrm{KCl}, 2.0 \mathrm{CaCl}_{2}, 1.0 \mathrm{MgCl}_{2}, 20 \mathrm{HEPES}$, and 10 glucose, $\mathrm{pH} 7.4$ (320-330 mOsm). For solutions with a $\mathrm{pH} \leq 6.0$, MES was used instead of HEPES for more reliable $\mathrm{pH}$ buffering (Chu et al., 2004, 2006; Jiang et al., 2009, 2010). A multibarrel perfusion system (SF-77, Warner Instrument) was used to achieve a rapid exchange of extracellular solutions.

ASIC1a channels were triggered by a drop in $\mathrm{pH}$ from 7.4 to given values (e.g., $\mathrm{pH}$ 6.0) every 2 min to allow a complete recovery of the channel from desensitization. During each experiment, a voltage step of $-10 \mathrm{mV}$ from the holding potential was applied periodically to monitor the cell capacitance and the access resistance. Recordings in which either the access resistance or the capacitance changed by $>10 \%$ during the experiment were excluded from data analysis. For $\mathrm{pH}$ activation curves, the ECF flowing out of one barrel of the perfusion system was $\mathrm{pH} 7.4$ while the ECF flowing out of the second barrel was switched to $\mathrm{pH} 7.0$, $6.9,6.8,6.5,6.3,6.0$, and 5.5 sequentially. Acid-triggered currents at each $\mathrm{pH}$ were normalized to the peak current activated at $\mathrm{pH}$ 5.5. Normalized values were fitted to the Hill equation using SigmaPlot 10 software to obtain $\mathrm{pH}_{50}$ values and Hill coefficients. To determine the time constant of the desensitizing portion of the ASIC currents, $\mathrm{pH}$ 6.0-activated cur-

\section{A $\mathrm{cHO-K1}$}

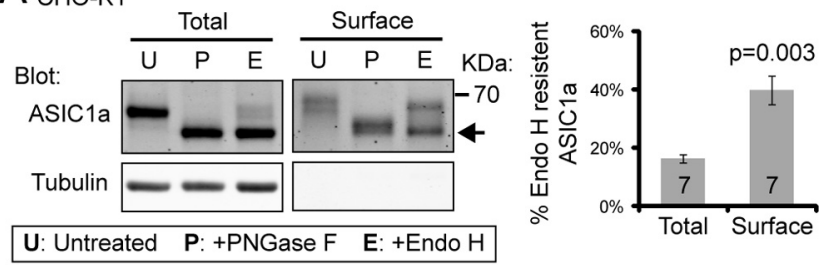

$\mathrm{B}$ in vivo (from P16 mice)

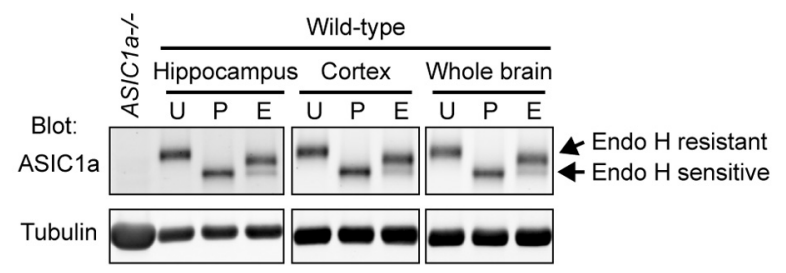

C Organotypic Hippocampal slices (P6 + 9-10DIV)
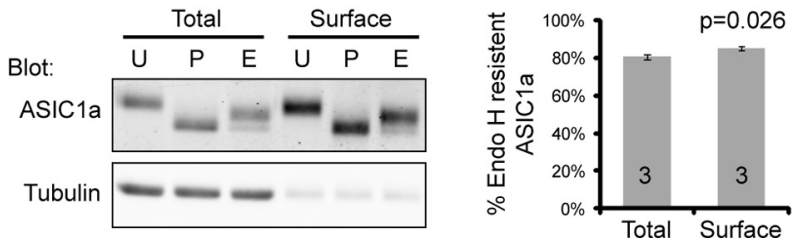

Figure 1. Most ASIC1a in the brain contains matured $N$-linked glycans. $\boldsymbol{A}$, Western blots and quantification showing ASIC1a glycosylation in $\mathrm{CHO}$ cells. CHO cells were transfected with ASIC1a and surface proteins were biotinylated $2 \mathrm{~d}$ after transfection. Cell lysates were untreated $(U)$, treated with PNGase $F(P)$ or endo $H(E)$. Total and surface proteins, isolated by NeutrAvidin precipitation, were blotted for ASIC1a and tubulin as indicated. Arrow indicates the endo $\mathrm{H}$-sensitive populations while the endo H-resistant population ran just below the $70 \mathrm{kDa}$ molecular weight marker. Right, Quantification of the percentage of endo H-resistant ASIC1a. $\boldsymbol{B}$, Western blots showing glycosylation of endogenous ASIC1a in hippocampus, cortex, or whole brain isolated from P16 mice. P16 mice were used here to match the age of hippocampal slices used in this study. Note that most ASIC1a in the brain was resistant to endo H. C, Western blots and quantification showing glycosylation of surface and total ASIC1a isolated from organotypic hippocampal slices. Note that the difference between surface $(85.2 \pm 1.1 \%)$ and total $(80.4 \pm 1.3 \%)$ fractions was small but significant statistically. Numbers on the bars indicate the total number of repeats (for $\boldsymbol{A}$ and $\boldsymbol{C}$ ); $n=4$ for $\boldsymbol{B} ; p$ values were from paired $t$ test.

rents were fitted by a single, standard exponential equation using Clampfit 10.2.

Expression and whole-cell electrophysiology in Xenopus oocytes. Culture and cDNA injection of Xenopus oocytes were done similar to what has been described previously (Collier and Snyder, 2009). Briefly, oocytes from albino Xenopus laevis were manually defolliculated following a $1 \mathrm{~h}$ treatment with $0.75 \mathrm{mg} / \mathrm{ml}$ type IV collagenase (Sigma-Aldrich) in $\mathrm{Ca}^{2+}$-free ND-96 containing the following (in $\mathrm{mm}$ ): $96 \mathrm{NaCl}, 2 \mathrm{KCl}, 1$ $\mathrm{MgCl}_{2}, 5$ HEPES, $\mathrm{pH}$ 7.4. Following nuclear injection of cDNAs $(0.06$ $\mu \mathrm{g} / \mu \mathrm{l}$ ) encoding WT or mutant ASICla, cells were incubated at $18^{\circ} \mathrm{C}$ for 20-24 h in modified Barth's saline containing the following (in $\mathrm{mM}$ ): 88 $\mathrm{NaCl}, 1 \mathrm{KCl}, 0.33 \mathrm{Ca}\left(\mathrm{NO}_{3}\right)_{2}, 0.41 \mathrm{CaCl}_{2}, 0.82 \mathrm{MgSO}_{4}, 2.4 \mathrm{NaHCO}_{3}, 10$ HEPES, $50 \mu \mathrm{g} / \mathrm{ml}$ gentamycin, $10 \mu \mathrm{g} / \mathrm{ml}$ sodium penicillin, $10 \mu \mathrm{g} / \mathrm{ml}$ streptomycin sulfate, $\mathrm{pH}$ 7.4. Oocytes were voltage-clamped (twoelectrode voltage-clamp) and currents were amplified with an Oocyte Clamp OC-725C (Warner Instruments), digitized with a MacLab/200 interface (ADInstruments), and recorded and analyzed with Chart software (ADInstruments). Recordings were performed at $-60 \mathrm{mV}$ in a Ringer's solution containing the following (in $\mathrm{mm}$ ): $116 \mathrm{NaCl}, 2 \mathrm{KCl}, 0.4$ $\mathrm{CaCl}_{2}, 1 \mathrm{MgCl}_{2}, 5$ HEPES, pH 7.4; $\mathrm{pH} 5$ solutions also contained 5 MES. ASIC currents were evoked by changing bathing solution from $\mathrm{pH} 7.4$ to pH 5.0 Ringer's. Mock or uninjected oocytes did not have $\mathrm{pH}$ 5.0-evoked currents.

Statistical analysis. For paired comparisons, we used a two-tailed Student's $t$ test and paired Wilconxon test unless otherwise indicated. For multiple comparisons, we used SAS 9.2 procedure GLM to perform the 


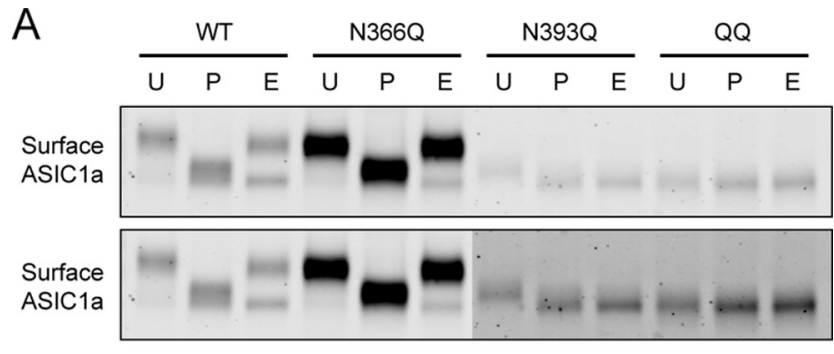

(Bottom panel: contrast adjusted to better show N393Q)

\begin{tabular}{|lll}
\hline U: Untreated & P: +PNGase $F$ & E: + Endo $H$ \\
\hline
\end{tabular}
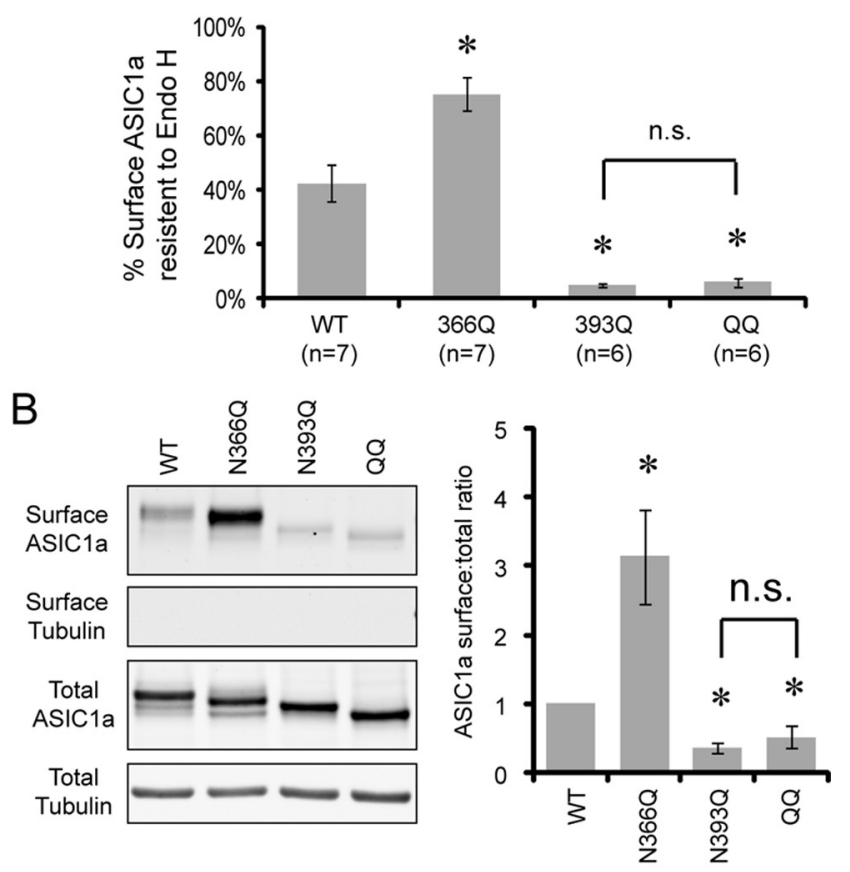

C

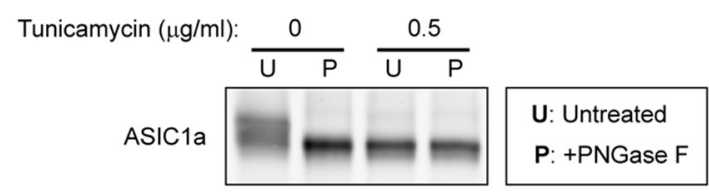

$\mathrm{D}$
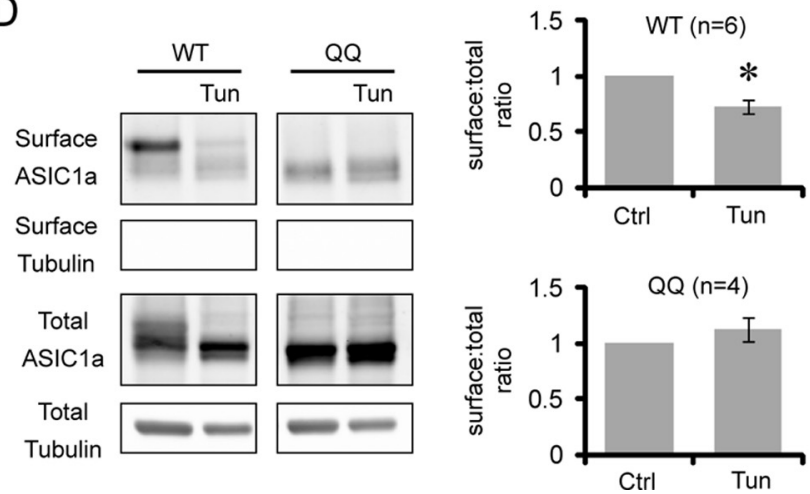

Figure 2. $\quad$-linked glycosylation regulates ASIC1a surface trafficking. $A$, Representative Western blot showing the sensitivity of WT ASIC1a, N366Q, N393Q, or QQ mutants to endo H and PNGase F treatment. CHO cells were transfected with different constructs as indicated. Surface proteins were biotinylated. Cell lysates were treated with PNGase $\mathrm{F}$ or endo $\mathrm{H}$ as described in Methods and Materials. Surface proteins were then isolated and analyzed by Western blot. PNGase F-treated samples all ran at the same position, indicating that the difference in migration patterns between untreated samples was due to differential glycosylation. Note that $\mathrm{N} 366 \mathrm{Q}$ increased the percentage of endo H-resistant populations. Also note the small shift in unbalanced ANOVA with a Bonferroni multiple-comparison adjustment, followed by pairwise $t$ test to check the difference between two groups. Data were reported as mean \pm SEM for the number of samples indicated.

\section{Results}

Fully glycosylated ASIC1a is preferentially trafficked to the cell surface

One key signature for protein maturation is the processing of $N$-linked glycans along the secretory pathway (Rotin et al., 2001; Helenius and Aebi, 2004). To assess glycosylation of ASIC1a, we transfected $\mathrm{CHO}$ cells with cDNAs encoding mouse ASIC1a, did surface biotinylation, and treated the cell lysates with endo $\mathrm{H}$ or PNGase F. Endo H removes immature $N$-linked glycans that have not undergone processing in the middle to late Golgi. In contrast, PNGase F removes both immature and mature $N$-linked glycans on proteins (Rotin et al., 2001; Helenius and Aebi, 2004). Similar to our previous results (Jing et al., 2011), $\sim 16 \pm 1 \%$ of total ASIC1a and $40 \pm 5 \%$ of surface ASICla were resistant to endo $\mathrm{H}$ treatment (Fig. 1A). These data imply that, at a steady-state level, while the $\mathrm{N}$-glycans on only a small fraction of intracellular ASIC1a in CHO cells has been processed through the middle to late Golgi, a full maturation facilitates an efficient surface expression of ASICla.

Next, we studied ASIC1a in the brain isolated from P16 mice, because this age matches that of the slices used in our in vitro studies below. We found that $77 \%$ of ASIC1a in the hippocampus was resistant to endo $\mathrm{H}$ treatment (Fig. $1 B$ ). Similarly, 72 and $77 \%$ of ASIC1a in the whole brain and cortex, respectively, contained mature $N$-linked glycans. These data showed that most $N$-glycans on ASICla in the brain have matured through middle to late Golgi.

To further assess glycosylation of the surface fraction of endogenous ASICla, we biotinylated organotypic hippocampal slices (P6 + 9-10 DIV) and performed similar Western blot analysis after endoglycosidase treatment. The percentage of endo $\mathrm{H}$-resistant ASICla at the surface was $85.2 \pm 1.1 \%$, slightly, but significantly ( $p=0.026$, paired $t$ test), higher than that of total ASIC1a $(80.4 \pm 1.3 \%)$ (Fig. $1 D)$. Together, our results indicate that $\mathrm{N}$-glycans on endogenous ASICla show a higher level of maturation than those on transfected ASICla in $\mathrm{CHO}$ cells. However, in both $\mathrm{CHO}$ cells and hippocampal slices, the surface fraction contained a higher percentage of fully glycosylated ASIC1a, suggesting that matured ASIC1a was preferentially trafficked to the cell surface in both systems.

\section{Glycosylation of Asn393 is critical for ASIC1a surface expression}

To further understand the mechanisms regulating ASIC1a glycosylation, we assessed the relative contribution of Asn366 and Asn393, the two potential glycosylation sites of mouse ASIC1a.

$\leftarrow$

migration between untreated and PNGase F-treated or endo H-treated N393Q, which shows that the majority of $\mathrm{N} 3930$ was glycosylated but was sensitive to endo $\mathrm{H}$ (the lower blot has contrast enhanced to better show this result of N3930). Bottom plot shows the quantification. Asterisks indicate significant differences from WT ( $p<0.001$, ANOVA). $\boldsymbol{B}$, Western blots and quantification (from 7 different repeats) showing the effect of Asn 366 and Asn 393 mutations on surface expression of ASIC1a. Asterisks indicate significant differences from WT ( $p<0.05$, Wilcoxon signed rank test). C, $\boldsymbol{D}$, Effect of tunicamycin on ASIC1a glycosylation ( $($ ) and surface trafficking (D). ASIC1a-transfected CHO cells were treated with $0.5 \mu \mathrm{g} / \mathrm{ml}$ tunicamycin for $24 \mathrm{~h}$. Untreated or PNGase F-treated lysates $(\boldsymbol{C})$ or surface and total fractions $(\boldsymbol{D})$ were analyzed by Western blot. Note that tunicamycin had no effect on surface/total ratio of $\mathrm{QQ}$, which has both glycosylation sites mutated. Asterisk indicates significant differences ( $p<0.01$, paired $t$ test) 

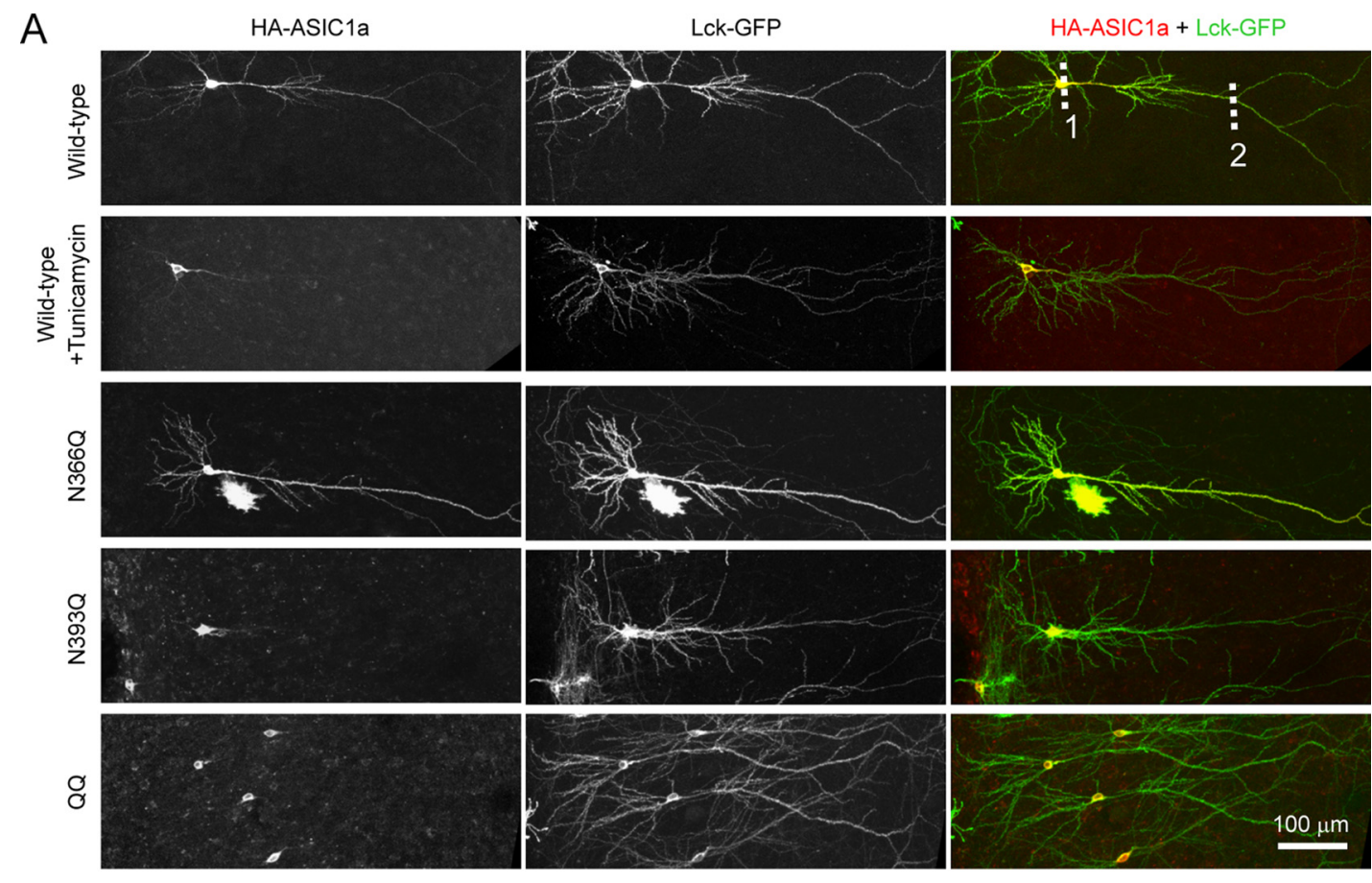

B

Line profile at the two positions illustrated in panel A
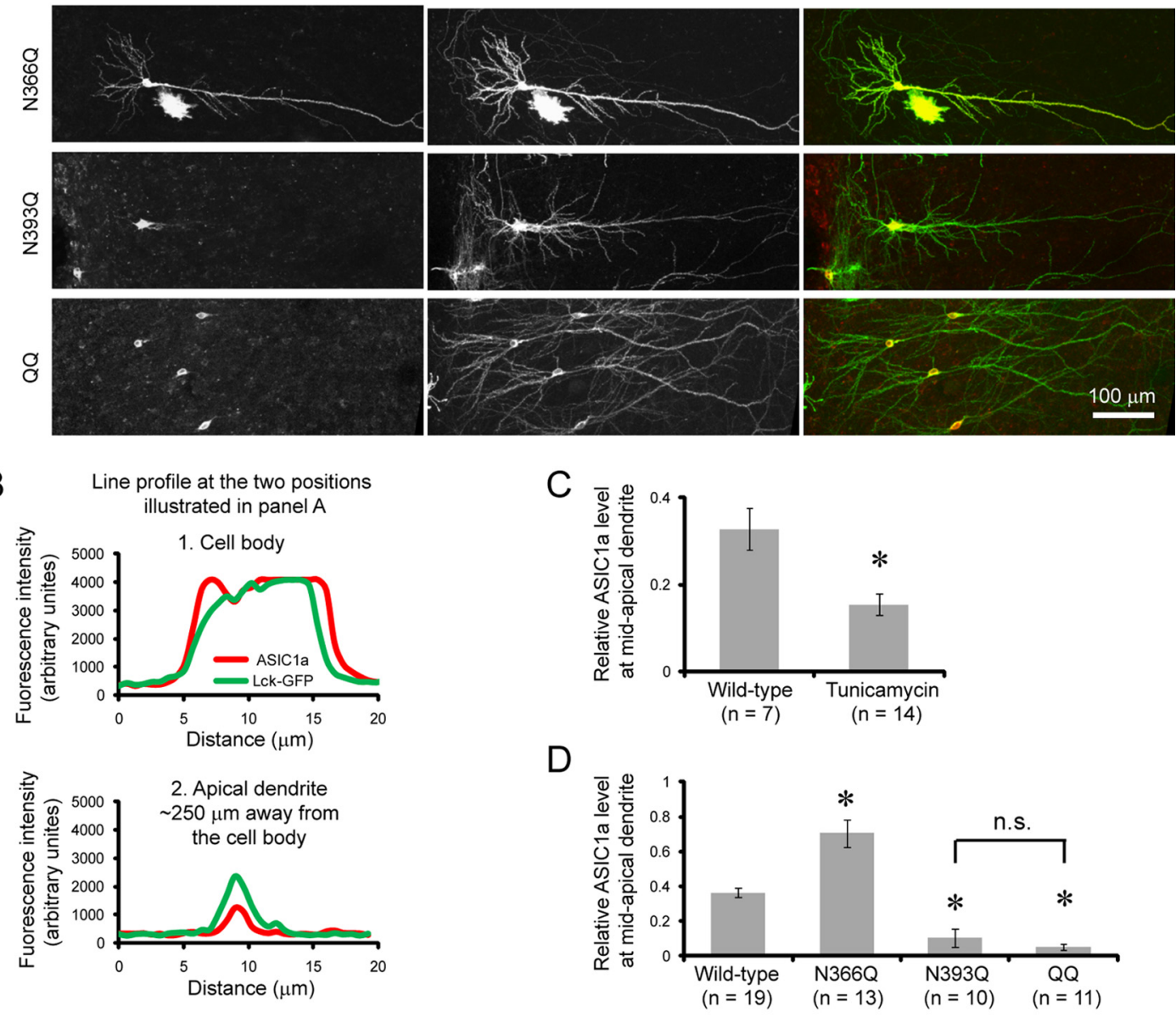

E
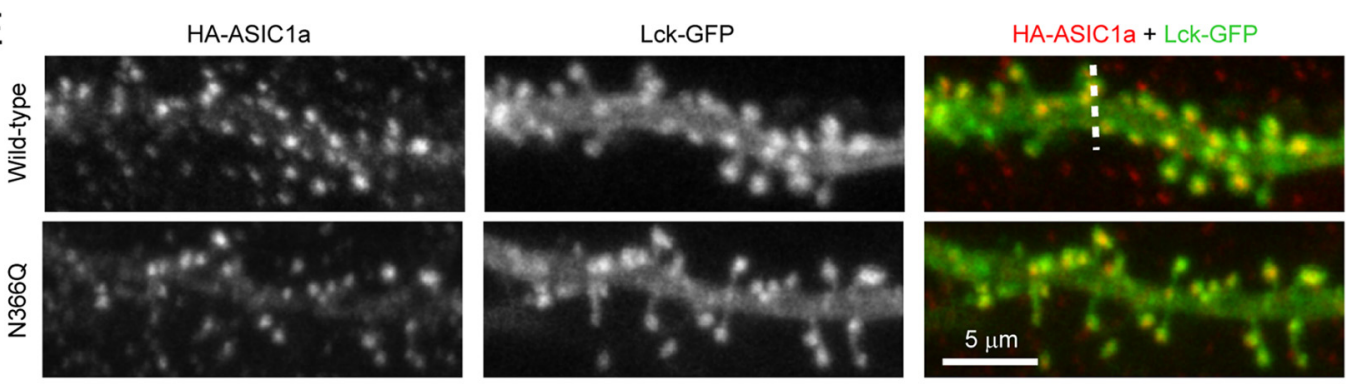

F
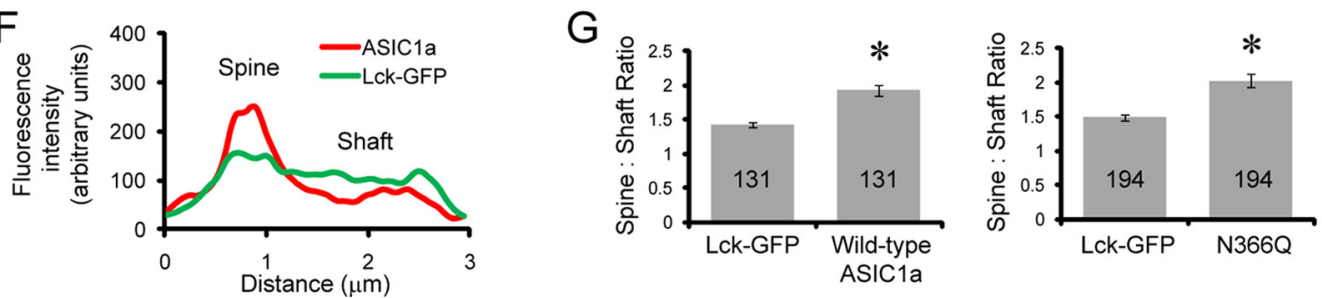

Figure 3. Differential effects of glycosylation mutants on dendritic targeting of ASIC1a. Hippocampal slices were cotransfected with HA-tagged wild-type ASIC1a, N366Q, N393Q, or QQ mutants together with a membrane targeted Lck-GFP (Benediktsson et al., 2005). Localization of transfected ASIC1a was visualized by immunofluorescence using an anti-HA (Figure legend continues.) 
We generated N366Q, N393Q, and QQ mutants and studied their effect on ASICla glycosylation. PNGase F-treated WT and single mutants ran at the same position as $\mathrm{QQ}$, showing that the difference in migration patterns between untreated WT and mutant ASICla was due to differential glycosylation (Fig. 2A). Untreated $\mathrm{N} 366 \mathrm{Q}$ ran slightly faster than WT, suggesting that modification of Asn366 led to a small change in molecular weight. Consistent with this speculation, untreated N393Q ran slightly slower than QQ or PNGase F-treated N393Q. These results show that both Asn366 and Asn393 were glycosylated but glycosylation of Asn366 only led to a small increase in the apparent molecular weight of ASIC1a. N366Q and N393Q also differed in the maturation of their $\mathrm{N}$-glycans. N366Q showed a significantly $(p<0.0001)$ higher percentage of endo H-resistant populations than the WT (Fig. 2A). However, most N393Q was sensitive to endo $\mathrm{H}$ treatment. These data indicate that glycans on Asn 393 but not those on Asn366 were preferentially processed in middle-to-late Golgi.

Next, we studied the effect of single and double Q mutants on ASIC1a surface expression. Compared with the WT, N366Q significantly $(p<0.0001)$ increased surface expression, while both N393Q and QQ mutants significantly $(p<0.05)$ reduced ASIC1a surface levels (Fig. $2 B$ ). The effect of N366Q is somewhat surprising because most mutants that disrupt $\mathrm{N}$-glycosylation either inhibit or have no effect on protein trafficking (Canessa et al., 1994; Quirk et al., 2004; Cai et al., 2005; Li et al., 2007; Vacca et al., 2011). These results indicate that maturation of Asn393 is necessary and sufficient for efficient surface expression of ASICla. In contrast, the reduced maturation and surface levels of N393Q suggest that processing of $\mathrm{N}$-glycans linked to Asn 366 may be a rate-limiting step for ASIC1a biogenesis.

The above results demonstrate a correlation between ASIC1a glycosylation and its surface expression. To directly test the effect of glycosylation, we treated the cells with tunicamycin, which inhibits the addition of core glycans (Prescher and Bertozzi, 2006). After $24 \mathrm{~h}$ in tunicamycin, almost all ASIC1a ran at the same position as PNGase F treatment samples, indicating a lack of $N$-linked glycans (Fig. 2C). As expected, tunicamycin significantly ( $p=0.002$, paired $t$ test) reduced ASICla surface expression by $27 \%$ (Fig. $2 D$ ). As an additional control, we studied the effect of tunicamycin on QQ and found that tunicamycin had no

\footnotetext{
$\leftarrow$

(Figure legend continued.) antibody. A, Representative confocal images showing the overall distribution of ASIC1a in transfected neurons. Left, ASIC1a localization. Middle, GFP fluorescence. Right, Merged image (HA immunofluorescence in red and GFP fluorescence in green). Note that WT and N3660 were present in most dendritic branches, with N3660 showing stronger dendritic staining in distal dendrites. Also note that the majority of tunicamycin-treated WT and almost all N393Q and QQ were restricted to the cell body. $\boldsymbol{B}$, Line profile showing the fluorescence intensity of ASIC1a (red line) and Lck-GFP (green line) along the two lines illustrated in A. C, Quantification of the effect of tunicamycin on relative ASIC1a levels at midapical dendrites. The ratio of dendritic to cell body ASIC1a fluorescence was normalized to that of Lck-GFP, which controls for passive diffusion of membrane proteins. Numbers in parenthesis indicate the number of neurons quantified from two different experiments. D, Quantification of the effect of glycosylation mutants on relative ASIC1a levels at midapical dendrites. Numbers in parenthesis indicate the number of neurons quantified from six (for WT), four (for N3660), and three (for N3930 and QQ) different experiments. Asterisks indicate significant differences from WT. $\boldsymbol{E}$, High-magnification view of a segment of apical dendrite. Note the presence of wild-type and N3660 ASIC1a in most dendritic spines. $F$, Line profile of the relative fluorescence intensity along the line illustrated in $\boldsymbol{E}$. Note the difference in relative ASIC1a/Lck-GFP fluorescence intensity in spine versus shaft. G, Quantification of Lck-GFP and ASIC1a spine/shaft ratio. Numbers on the bars indicate the total number of spines quantified from 9 wild-type and 12 N3660 transfected neurons from three separate experiments. Asterisks indicate significant differences from Lck-GFP ( $p<0.0001$, Student's t test).
}
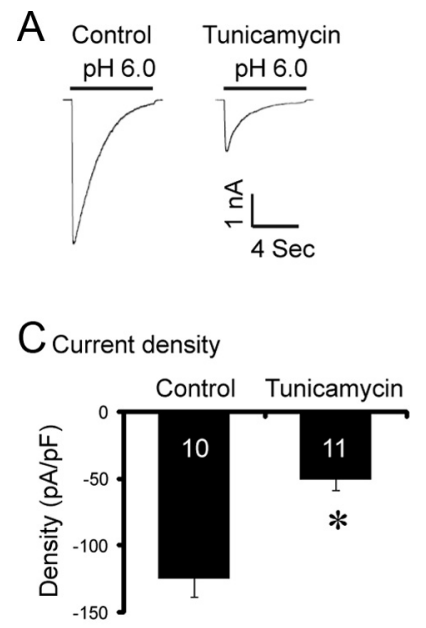

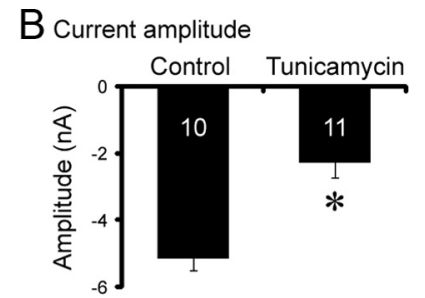

$\mathrm{D}$ Desensitization rate

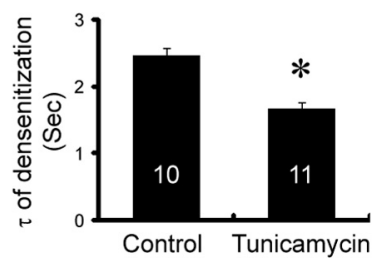

Figure 4. Tunicamycin reduces ASIC1a current density. CHO cells were transfected with ASIC1a and treated with DMSO (control) or $0.5 \mu \mathrm{g} / \mathrm{ml}$ tunicamycin for $24 \mathrm{~h}$. $\boldsymbol{A}-\boldsymbol{D}$, Representative traces $(\boldsymbol{A})$ and quantification showing the effect of tunicamycin on the amplitude $(\boldsymbol{B})$, density $(\boldsymbol{C})$, and rate of desensitization (D) of pH 6-activated current. Asterisks indicate significant $(p<0.001$, paired $t$ test) differences. Numbers on the bars indicate total number of cells recorded.

effect ( $p=0.17$, paired $t$ test) on surface expression of QQ (Fig. $2 D$ ). These results showed that $N$-glycosylation is necessary for efficient surface trafficking of ASICla.

\section{Glycosylation on Asn393 regulates dendritic targeting of ASIC1a}

In addition to surface expression, subcellular targeting is another key aspect of trafficking in neurons. In our previous studies, we showed that ASIC1a is preferentially targeted to dendrites (Zha et al., 2006, 2009b). To test the hypothesis that glycosylation is required for ASICla dendritic targeting, we transfected organotypic hippocampal slices with HA-ASICla together with a membrane-targeted Lck-GFP (Benediktsson et al., 2005), which facilitates the visualization of transfected neurons. We used $\mathrm{N}$-terminal HA-tagged cDNA constructs because N-terminal HA-tagged ASICla shows a localization pattern similar to that of endogenous proteins (Zha et al., 2006, 2009b). Similar to our previous studies (Zha et al., 2006, 2009b), wild-type ASICla was present in most dendrites (Fig. 3A). To quantitatively analyze the dendritic localization of ASICla, we measured the relative ASIC1a immunofluorescence intensity at the cell body and, in the middle segment of the apical dendrite, at $\sim 250 \mu \mathrm{m}$ away from the cell body (Fig. $3 A, B$ ). As a control for diffusion of membrane proteins, we normalized dendrite/cell body ratio of ASICla immunofluorescence to that of Lck-GFP. Tunicamycin treatment significantly ( $p=0.0096$, Student's $t$ test) reduced the level of ASICla in dendrites, suggesting that $\mathrm{N}$-glycosylation was required for efficient dendritic trafficking of ASICla (Fig. 3A,C).

Since N366Q increased ASIC1a glycosylation while N393Q had the opposite effect (Fig. 2A), we wondered whether they differentially affect dendritic targeting of ASIC1a in neurons. Compared with WT ASIC1a, N366Q showed a similar pattern of distribution but had significantly $(p<0.001$, ANOVA) increased levels in the dendrite (Fig. $3 A, D$ ). In contrast, N393Q and QQ exhibited diminished localization in dendrites. These data show that maturation of Asn393-linked glycans is required for dendritic targeting of ASICla, and the level of ASICla in dendrites correlates with its level of maturation. 
Next, we asked whether N366Q affects the targeting to dendritic spines. We quantified the fluorescence intensity of ASIC1a and Lck-GFP at spine heads and on the dendritic shaft (Fig. 3E-G). The spine/shaft ratio of ASICla fluorescence was significantly ( $p<0.0001$, Student's $t$ test) higher than that of the membranetargeted Lck-GFP. This result demonstrates that ASIC1a is preferentially targeted to dendritic spines. N366Q showed a similar level of enrichment in dendritic spines. Since N366Q had increased dendritic levels, an unchanged spine/shaft ratio from the wild type indicates that absolute level of N366Q in spines is higher than WT.

\section{Glycosylation of ASIC1a regulates its current properties}

As a first step to address the functional significance of ASIC1a glycosylation, we studied $\mathrm{pH}$-activated current properties in CHO cells. To directly test whether glycosylation of ASIC1a regulates its current properties, we treated $\mathrm{CHO}$ cells with or without tunicamycin for $24 \mathrm{~h}$. Application of acidic $\mathrm{pH}$ induced a fast desensitizing inward current, characteristic of ASIC1a (Fig. $4 A)$. Tunicamycin treatment significantly $(p<0.001$, paired $t$ test $)$ reduced acidactivated current amplitude and density, increased the rate of desensitization of ASICla (Fig. $4 B-D$ ), but had no effect on $\mathrm{pH}$ sensitivity. $\mathrm{pH}_{50}$ for control and tunicamycintreated cells were $6.42 \pm 0.03$ and $6.38 \pm$ 0.03 , respectively ( $n=5$ for both control and tunicamycin-treated cells, $p=0.428$, paired $t$ test). These results are consistent with our biochemical analysis, which showed that tunicamycin reduced ASICla surface levels.

To gain more insight into the mechanism, we studied $\mathrm{pH}$-activated current of the glycosylation mutants of ASICla. N366Q increased the amplitude, density, and $\mathrm{pH}$ sensitivity (Fig. 5A-D). The $\mathrm{pH}_{50}$ for N366Q was 6.82 as opposed to 6.30 for WT $(p<0.001$, Student's $t$ test). In contrast, N393Q and QQ did not show a change in $\mathrm{pH}$ sensitivity but had significantly reduced $\mathrm{pH}$-activated current. In addition, the rate of desensitization was significantly ( $p<0.01$, Student's $t$ test) changed in all mutants (Fig. $5 E$ ); $\tau$ of desensitization (in seconds) was as follows: WT, $2.68 \pm 0.34 ; \mathrm{N} 366 \mathrm{Q}, 5.73 \pm 0.82 ; \mathrm{N} 393 \mathrm{Q}, 1.85 \pm 0.21$; QQ, $1.35 \pm 0.08$. These results show that the maturation of Asn393 is important for ASICla channel function.

The reduced current of N393Q raised a question of whether N393Q can function as a dominant-negative construct. To answer this question, we coexpressed wild-type ASIC1a and the N393Q mutant in CHO cells and studied pH 6-activated current. Cells expressing both N393Q and wild-type ASIC1a had significantly ( $p<0.001$, paired $t$ test) reduced current density and faster $\tau$

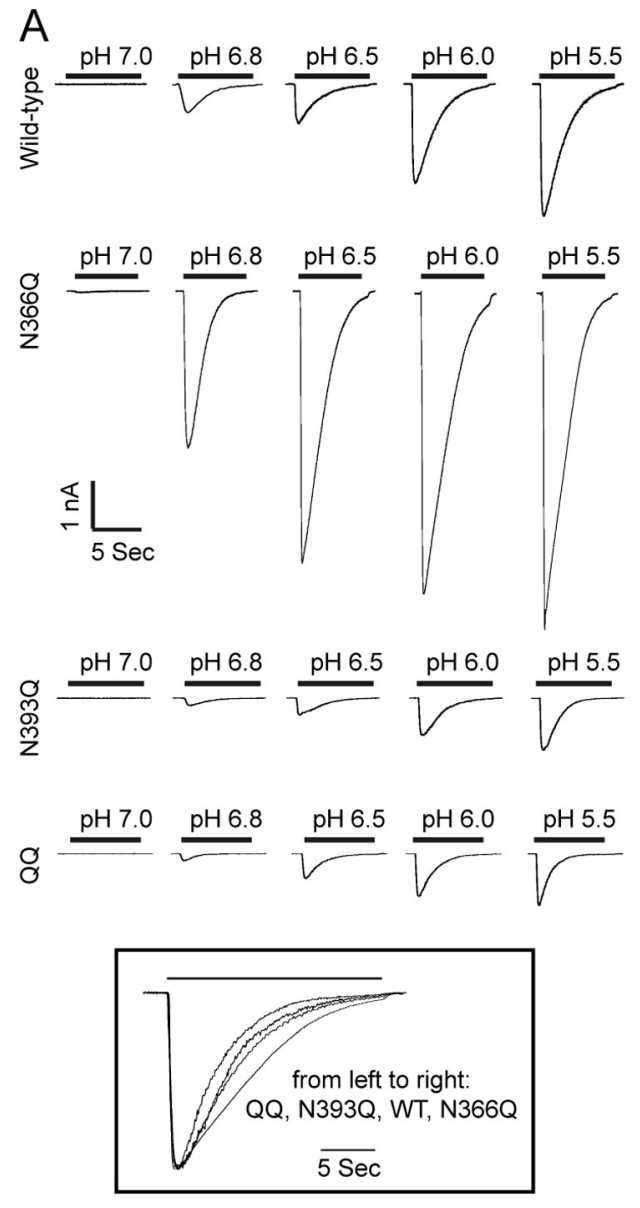

B pH sensitivity

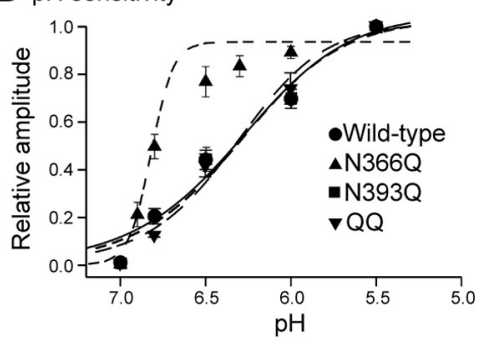

C Current amplitude

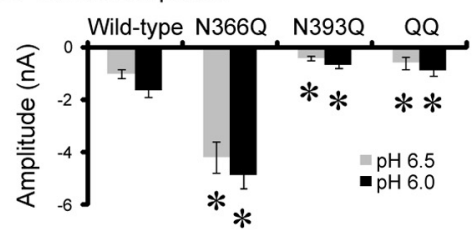

D current density

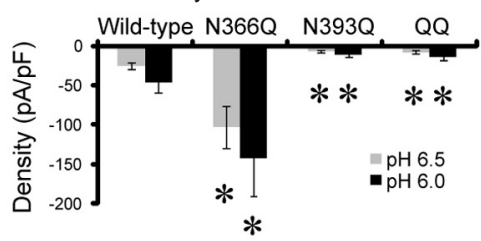

E Desensitization rate

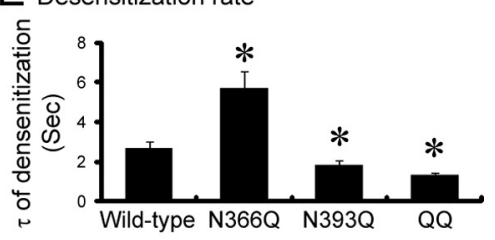

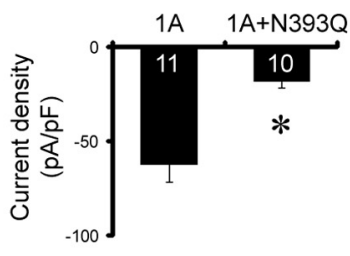

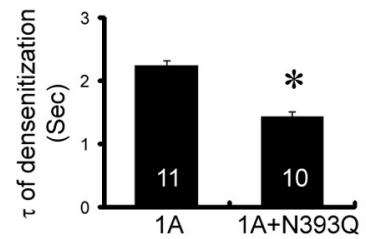

Figure 5. Effects of glycosylation mutants on ASIC1a current properties. CHO cells were transfected with wild-type or mutant ASIC1a as indicated. $A$, Representative $\mathrm{pH}$-induced current from cells expressing wild-type or mutant ASIC1a. Each set came from the same transfected cell. Inset at the bottom shows superimposed pH 6 currents for WT and mutant ASIC1a to illustrate the differences in the rate of desensitization. $\boldsymbol{B}$, pH sensitivity of wild-type and mutant $\mathrm{ASIC1a}$. The values for $\mathrm{pH}_{50}$ were as follows: WT, $6.30 \pm 0.19(n=8) ; \mathrm{N} 3660,6.82 \pm 0.03(n=6) ; \mathrm{N} 3930,6.31 \pm 0.25(n=10) ; Q Q, 6.32 \pm 0.12(n=5)$. C, D, Quantification of current amplitude $(\boldsymbol{C})$ and density (D) of pH 6.5-induced and pH 6.0-induced current. $\boldsymbol{E}$, Quantification of the rate of desensitization of pH 6-activated current. $\boldsymbol{F}$, Typical traces and quantifications showing the effect of N3930 coexpression on wild-type ASIC1a current. Numbers on the bars indicate the number of cells quantified. Asterisks indicate significant differences from wild-type ASIC1a ( $p<0.01$, Student's $t$ test).

of desensitization, both similar to when N393Q was expressed alone (Fig. $5 F$ ). These data demonstrate that N393Q functions dominantnegatively in inhibiting ASICla current.

Our results on N366Q contrast with those of a previous report, in which an N366A mutant reduced ASIC1a current amplitude in Xenopus oocytes (Kadurin et al., 2008). To test whether the discrepancy was due to the specific mutation (Asn $\rightarrow$ Gln vs Asn $\rightarrow$ Ala), we generated an ASIC1a N366A mutant and found that it also increased surface expression and current amplitude of ASIC1a (Fig. 6A,B). To further test whether the discrepancy arose from the difference in the culture system, we studied acidactivated current in Xenopus oocytes and found that N366A again 
A mouse ASIC1a ( $\mathrm{CHO}$ cells)
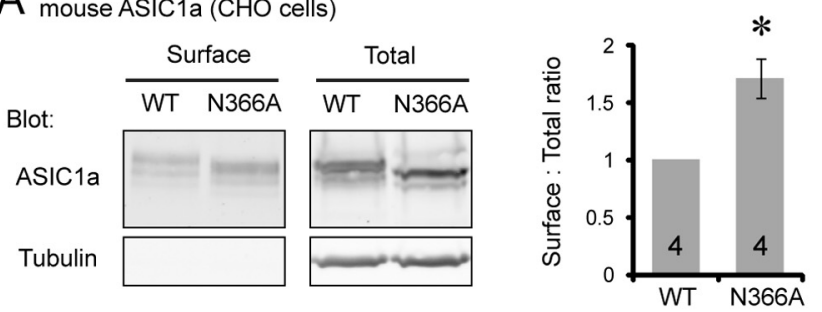

B mouse ASIC1a ( $\mathrm{CHO}$ cells)
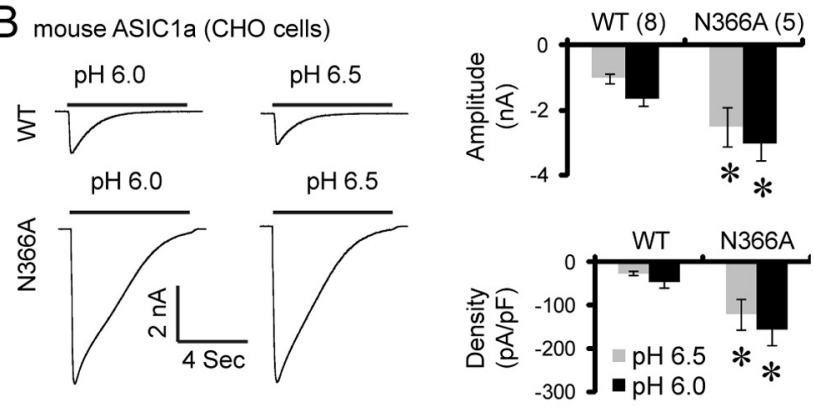

C mouse ASIC1a (Xenopus oocyte)

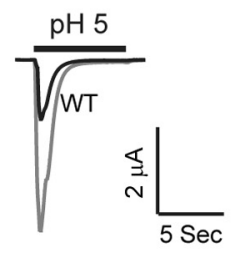

N366A

$\mathrm{D}$ rat ASIC1a (CHO cells)

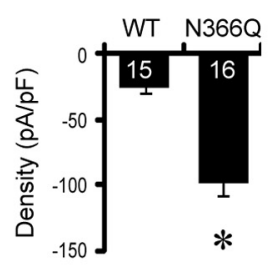

$\mathrm{F}$ human ASIC1a ( $\mathrm{CHO}$ cells)

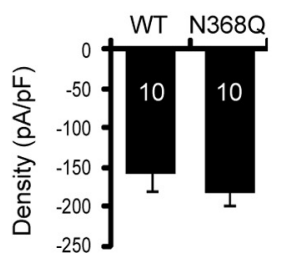

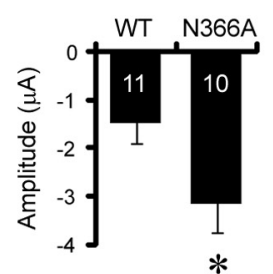

$E_{\text {rat ASIC1a (oocyte) }}$

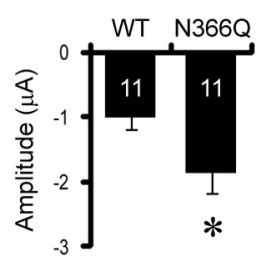

G human ASIC1a (oocyte)

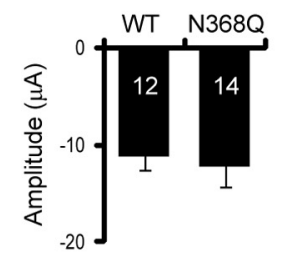

Figure 6. The effect of Asn 366 mutation is species-dependent. $A$, Western blot and quantification of surface/total levels of wild-type and N366A mouse ASIC1a. $\boldsymbol{B}-\boldsymbol{G}$, Typical current and quantification of $\mathrm{pH}$-activated current in $\mathrm{CHO}(\boldsymbol{B}, \boldsymbol{D}, \boldsymbol{F})$ and Xenopus oocytes $(\boldsymbol{C}, \boldsymbol{E}, \boldsymbol{G})$ expressing mouse $(\boldsymbol{B}, \boldsymbol{C})$, rat $(\boldsymbol{D}, \boldsymbol{E})$, or human $(\boldsymbol{F}, \boldsymbol{G}) \mathrm{ASIC}$ 1a. Numbers in parentheses or on the bars indicate total number of repeats (for $\boldsymbol{A}$ ) or total number of cells or oocytes analyzed. Asterisks indicate significant differences from wild type ( $p<0.05$, Student's $t$ test).

increased current amplitude in oocytes (Fig. 6C). Another difference between the two studies is that we used mouse ASICla while Kadurin et al. (2008) used rat ASIC1a. We therefore asked whether the N366Q mutation has differential effects on ASIC1a from different species. We generated the same mutation in rat and human ASIC1a and analyzed their current in both $\mathrm{CHO}$ cells and Xenopus oocytes. N366Q in rat ASICla also increased current density (in CHO cells) or amplitude (in oocytes) (Fig. 6D,E). In contrast, an N368Q mutation in human ASIC1a (equivalent to N366Q in mouse and rat ASIC1a) had no effect on ASICla current amplitude (Fig. $6 F, G$ ). These data suggest that the effect of $\mathrm{N} 366 \mathrm{Q}$ is species-dependent, although we still do not know the reason for the discrepancy between the previous (on rat ASIC1a) and current study. Nevertheless, our electrophysiology recording in $\mathrm{CHO}$ and oocytes are consistent with our biochemical analysis on surface expression. Moreover, our functional results on acidosis-induced spine loss in organotypic hippocampal slices (see below and Fig. 8) also support an increased pH sensitivity and/or current of N366Q.

\section{Acidosis reduces spine density and length via ASIC1a}

The dendritic presence of ASICla suggests the hypothesis that acidosis has a specific effect at the dendritic region. This hypothesis is interesting because current literature has mainly studied acidosis on neuronal survival (Immke and McCleskey, 2001; Yermolaieva et al., 2004; Sherwood and Askwith, 2008, 2009; Duan et al., 2011). However, a site-specific effect at the dendritic region is critical functionally because dendritic and/or spine remodeling plays a key role in regulating neuron physiology and is associated with changes in plasticity (Bhatt et al., 2009). To test the above hypothesis, we transfected organotypic hippocampal slices with Lck-GFP and treated the slices for $1 \mathrm{~h}$ with a $\mathrm{pH} 7.4$ or a $\mathrm{pH} 6.0$ medium. Somewhat surprisingly, $1 \mathrm{~h} \mathrm{pH} 6.0$ treatment had no apparent effect on dendrites (Fig. 7A). Since enlargement or "blebbing" of dendritic shafts was a typical phenomenon observed during dendrotoxicity (Oliva et al., 2002; Chen et al., 2011), we further quantified the shaft width of apical dendrites and found that $\mathrm{pH} 6$ treatment had no significant effect on the width of dendritic shaft (Fig. 7A).

Next, we analyzed dendritic spines and found that $\mathrm{pH} 6.0$ reduced spine density in a time-dependent manner (Fig. $7 B$ ); 30 min treatment had no significant effect on spine density while $60 \mathrm{~min}$ and $90 \mathrm{~min}$ treatment led to a 21 and $31 \%$ reduction, respectively. Interestingly, at 30 and $60 \mathrm{~min}$, but not 90 min, $\mathrm{pH} 6$ also reduced the average length of dendritic spines. Since ASIC1a is the primary postsynaptic proton receptor in brain neurons (Zha et al., 2006), we further asked whether the effect was mediated by ASIC1a. Deleting ASIC1a abolished pH 6 -induced reduction in spine density and length (Fig. 7C). Indeed, $\mathrm{pH} 6$ further led to a marginally significant $(p=0.032$, one-tailed $t$ test) increase of spine density in $\mathrm{ASICla}^{-1-}$ slice neurons (Fig. $7 C$ ). These results demonstrate that acidosis reduces spine density and length in a time- and ASICladependent manner.

N366Q and N393Q changed glycosylation and dendritic targeting, and N366Q also increased pH sensitivity of ASICla. Therefore, we speculated that these mutants would affect acidosis-induced spine loss. We transfected hippocampal slices with wild-type ASIC1a, N366Q, or N393Q mutant together with Lck-GFP, and treated the slices with acidic media. Neurons expressing wild-type ASIC1a showed a $24 \%$ spine loss and a $13 \%$ reduction in spine length in response to a $1 \mathrm{~h} \mathrm{pH} 6$ treatment, while pH 6.5 had no significant effect (Fig. $8 A-C$ ). To better compare the effect of ASIC1a overexpression to that of endogenous ASIC1a, we analyzed neurons transfected with empty vector or wild-type mouse ASICla. We found that $\mathrm{pH} 6$ reduced spine density and spine length to an extent similar to that in neurons expressing empty vector or mouse ASICla (Fig. $8 D$ ). In contrast, $\mathrm{pH} 6$ reduced spine density of N366Q-transfected neurons by $36 \%$, significantly higher than that of WT ASIC1a ( $p=0.0347$, pairwise $t$ test). Consistent with an increased $\mathrm{pH}$ 
sensitivity, N366Q-expressing neurons showed a $26 \%$ spine loss at $\mathrm{pH} 6.5$, significantly higher than that of WT ASIC1a ( $p=0.0105$, pairwise $t$ test). Since N393Q was restricted to the cell body and reduced $\mathrm{pH}$-activated current in a dominant-negative manner, we speculated that N393Q would abolish the effects of acidosis. Indeed, acidic media did not reduce spine density of N393Q-transfected neurons. Rather, both pH 6.5 and $\mathrm{pH} 6$ increased spine density of N393Q-expressing neurons, a result consistent with our data in ASIC $1 a^{-1-}$ slices (see Fig. $7 C$ ). In addition to the change in spine density, spine length was also affected by N366Q and N393Q. Table 1 presents a summary of these data. Together, our results suggest that maturation of ASIC1a plays a critical role in regulating acidosis-induced spine remodeling.

\section{Discussion}

Our results revealed two major findings concerning ASIC1a regulation and function. First, we demonstrated that acidosis reduced spine density and length in an ASICla-dependent manner. This finding extends the current knowledge concerning acidosis in neuronal injury and uncovers a novel link between acidosis and changes in synaptic connectivity. Second, our data tied ASICla glycosylation with its trafficking. Not only are these processes pivotal to ASICla biogenesis, our results further emphasize the importance of these processes in regulating ASICla channel properties and acidosis-induced spine remodeling. These data highlight the importance of ASICla glycosylation and acidosis in regulating neural plasticity.

\section{Regulation of ASIC1a glycosylation}

It is interesting that most ASICla in the brain contained $\mathrm{N}$-linked glycans that were endo $\mathrm{H}$-resistant. This observation contrasts with our results in $\mathrm{CHO}$ cells and a previous study in Xenopus oocytes (Kadurin et al., 2008), where the majority of ASIC1a was immature. Nevertheless, in both in vivo and in vitro systems, matured ASIC1a was preferentially inserted into the cell membrane. These data indicate that regulating protein maturation is an efficient way to control ASIC1a function.

It is also interesting to observe that the two glycosylation sites on ASICla had opposite effects. While maturation of Asn393 was required for ASICla biogenesis, glycosylation of Asn366 was dispensable. Indeed, mutating Asn366 increased the percentage of endo $\mathrm{H}$-resistant ASIC1a, suggesting that the maturation of $\mathrm{N}$-glycans linked to Asn366 is an inefficient process during ASICla biogenesis. The exact mechanism for this phenomenon is unclear. However, Asn366 is partially buried in the crystal structure (Jasti et al., 2007;
A
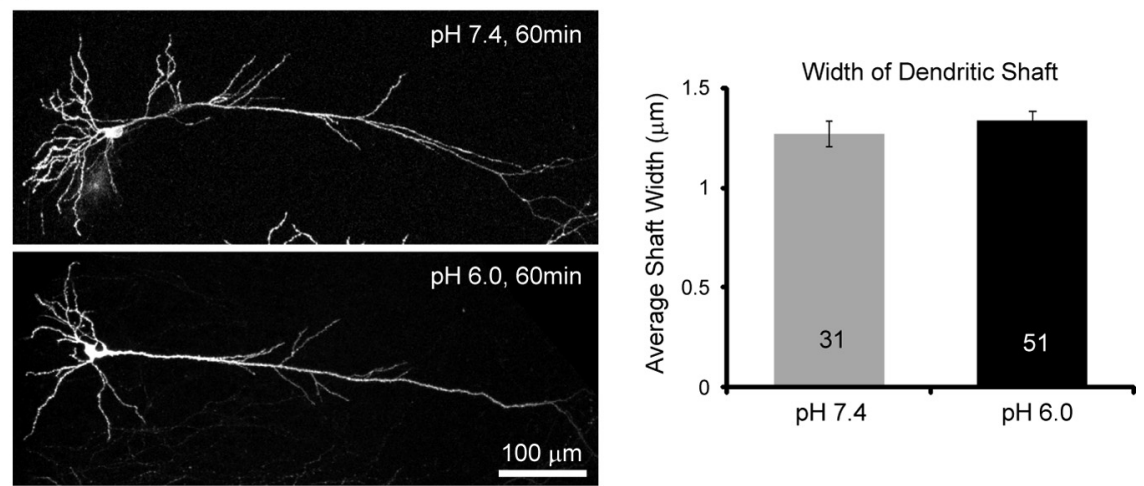

$\mathrm{pH} 7.4$ $\mathrm{pH} 6.0$

C

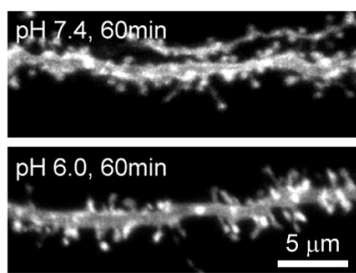

Spine Density

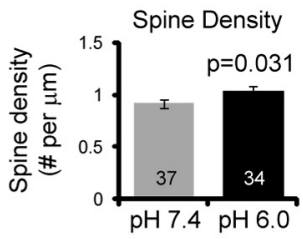

$\mathrm{pH} 7.4$
$\mathrm{pH} 6.0$

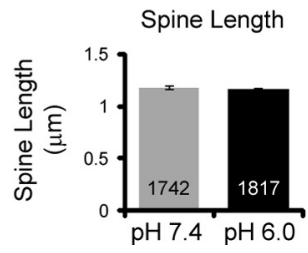

Figure 7. Chronic acidosis reduces spine density and length in an ASIC1a-dependent manner. $\boldsymbol{A}-\boldsymbol{C}$, Hippocampal slices from P5-P6 wild-type $(\boldsymbol{A}, \boldsymbol{B})$ or $A$ SIC1a ${ }^{-/-}(\boldsymbol{C})$ mice were transfected with Lck-GFP at 8-9 d in culture. Two days after transfection, slices were treated with a pH 7.4 or pH 6.0 medium for different time as indicated. $\boldsymbol{A}$, Left, Representative images showing the overall view of a CA1 neuron incubated for $60 \mathrm{~min}$ in pH 7.4 or pH 6.0 medium. Right, Quantification showing the average width of a segment of apical dendrites of CA1 neurons. No significant difference was seen between $\mathrm{pH}$ 7.4-treated and pH 6.0-treated neurons; $p=0.417$, Student's $t$ test. $B$, $C$, Top, High-magnification view of a segment of apical dendrite from wild-type $(\boldsymbol{B})$ or $\mathrm{ASIC1a^{-/- }}$ (C) slices after $60 \mathrm{~min}$ of pH 7.4 or pH 6.0 treatment. Bar graphs below show the quantification of spine density and length. Dashed lines in $\boldsymbol{B}$ indicate that data from the three time points were from different sets of experiments. Numbers on the bars indicate the total number of neurons (for density) or spines (for length) quantified from four to five (wild type) or three $\left(\mathrm{ASIC} \mathrm{Ca}^{-1-}\right)$ separate experiments. Asterisks indicate significant differences from the corresponding pH 7.4 controls ( $p<0.01$, Student's $t$ test). The $p$ value on spine density of $A S I C 1 a^{-1-}$ neurons was obtained from one-tailed $t$ test.

Gonzales et al., 2009), which suggests that glycans on this residue may not be processed efficiently and thus may increase the retention of ASIC1a early in the secretory pathway. This is a critical point, because it suggests a potential layer of ASIC1a regulation in the ER. It will be of future interest to test whether ER stress affects the maturation process of ASIC1a. Of note, ASIC1a contributes to multiple diseases, including stroke, multiple sclerosis, and seizures; all generate ER stress (Xiong et al., 2004; Friese et al., 2007; Ziemann et al., 2008). Thus, answering the above question will provide important insights toward a better understanding of how acidosis and ASICla contribute to diseases. 
A

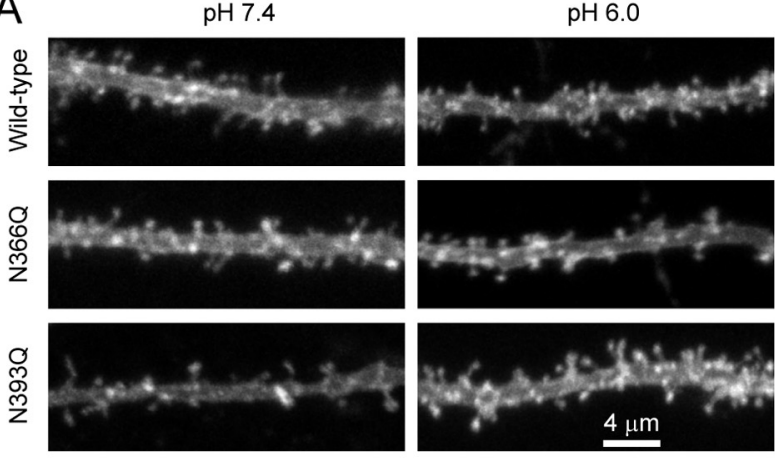

B

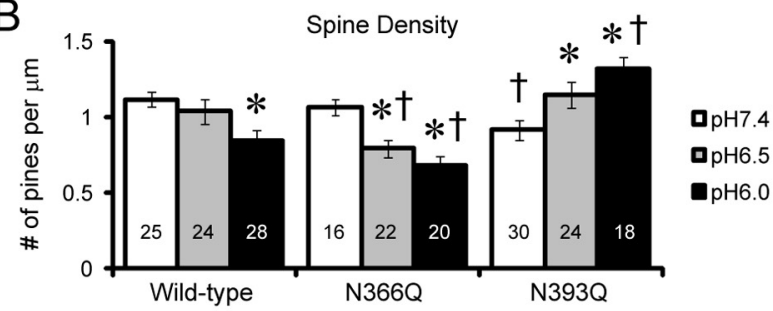

C

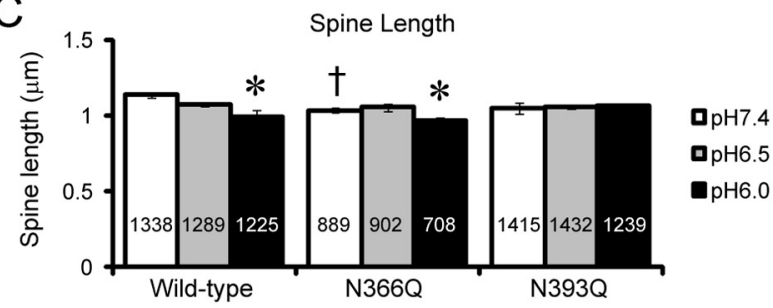

D vector vs. ASIC1a
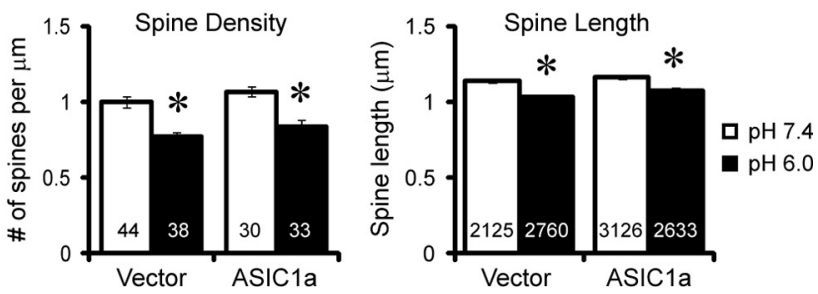

Figure 8. N3660 and N3930 have opposite effect on acidosis-induced spine loss. $\boldsymbol{A}$, Representative images showing a segment of an apical dendrite from CA1 neurons transfected with the indicated construct and treated for $1 \mathrm{~h}$ with a pH 7.4 or pH 6.0 medium. B, C, Quantification showing average spine density $(\boldsymbol{B})$ or spine length ( $\boldsymbol{C}$. D, Quantification showing pH 6-induced changes in spine density and length in neurons transfected with vector only or a mouse ASIC1a expression construct. Numbers on the bars indicate the total number of neurons (for density) or spines (for length) quantified from four separate experiments. Asterisks indicate significant difference from $\mathrm{pH} 7.4$ of the corresponding construct while daggers indicate significant difference from wild-type ASIC1a ( $p<0.05$, ANOVA).

Table 1. Summary of pH 6-induced spine remodeling

\begin{tabular}{lccccc}
\hline & Endogenous ASIC1a & Overexpression & N3660 & N3930 & ASIC1a knockout \\
\hline Spine density & $\downarrow$ & $\downarrow$ & $\downarrow \downarrow$ & $\uparrow \uparrow$ & $\uparrow$ \\
Spine length & $\downarrow$ & $\downarrow$ & $\downarrow$ & $\leftrightarrow$ & $\leftrightarrow$ \\
\hline
\end{tabular}

Note that the direction of changes with ASIC1a and N3660 overexpression was the same as that of endogenous ASIC1a. In contrast, the changes in N393Q-expressing neurons were in the same direction as those in ASIC1a knockout neurons.

\section{ASIC1a in dendritic spines}

Targeting to different neuronal compartments is a fundamental process that regulates the functional outcome of an ion channel. Using an EGFP to mark transfected neurons, we previously showed that ASICla is present in most dendritic spines (Zha et al., 2006, 2009b). However, a membrane protein may show a higher intensity in spines than a soluble GFP. Thus, it remains questionable whether ASIC1a was preferentially enriched in spines. We found here that the relative level of ASIC1a in spines was significantly higher than that of the membrane-anchored Lck-GFP. This result is consistent with previous studies showing an enrichment of ASIC1a in synaptosomal preparation (Wemmie et al., 2002; Zha et al., 2009b), and demonstrates clearly that ASIC1a is preferentially trafficked to dendritic spines.

As suggested by its localization, ASIC1a regulates spine remodeling. Knocking down ASIC1a or expressing a dominantnegative ASIC1a reduces spine density (Zha et al., 2006). Consistent with these data, expressing N393Q, which reduced surface expression, dendritic targeting, and acid-induced current, decreased spine density in control pH 7.4 conditions. On the other hand, ASIC1a overexpression increased spine density in our previous work but did not have significant effect on spine density here. The reason for this discrepancy is unclear, but possibly stems from the difference in species used. In our earlier study, we used human ASIC1a in transient transfection, or transgenic mice carrying a human ASIC1a transgene (Zha et al., 2006). Here, we used mouse ASIC1a, which has drastically $(\sim 10$-fold $)$ reduced current compared with human ASIC1a (Fig. $6 \mathrm{~B}$ vs $6 \mathrm{~F}$; $6 C$ vs $6 G$ ). Thus, it is possible that overexpressing mouse ASIC1a may not lead to as dramatic a change in acid-activated responses as human ASICla. Another possible explanation is the age of slices. For organotypic rodent hippocampal slices, spine density is $\sim 0.5-0.8 / \mu \mathrm{m}$ in cultures of $\leq 2$ weeks and reaches a plateau of $1.0-1.2 / \mu \mathrm{m}$ at $\sim 2-3$ weeks in culture (Collin et al., 1997; De Simoni et al., 2003). In our previous studies, we used P13-P14 slices, which are relatively immature (Zha et al., 2006, 2009b). In contrast, the P17 slices used here had a spine density of 1.0-1.1/ $\mu \mathrm{m}$, which reached or was close to the plateau value of a mature culture (Collin et al., 1997; De Simoni et al., 2003). It is possible that this developmental change also contributes to the lack of an effect of ASIC1a overexpression. Although the exact mechanism remains to be clarified in future studies, our results suggest that multiple factors contribute to the effects of ASIC1a on spine remodeling.

\section{Acidosis and remodeling in dendritic region}

While it has been known for some time that ASICs localize to dendrites and mediate proton-induced increase in $\left[\mathrm{Ca}^{2+}\right]_{\mathrm{i}}$ there (Wemmie et al., 2002; Alvarez de la Rosa et al., 2003; Zha et al., 2006, 2009b), no study has addressed a potential site-specific effect of acidosis in the dendritic region. Our finding that $1 \mathrm{~h}$ of pH 6.0 treatment had no obvious effect on dendritic architecture is somewhat unexpected, given that dendrites are sensitive to excitotoxic stimuli (Oliva et al., 2002; Chen et al., 2011). Nevertheless, this result is consistent with one electron microscope study showing that there was no major morphological changes in dendrites after $1-1.5 \mathrm{~h}$ of respiratory acidosis, which decreased brain pH to 6.3-6.0 (Schlote et al., 1975).

Although acidosis had no major effect on dendrites, it reduced spine number. We speculate that this effect is due to increased excitotoxicity resulting from ASICla activation; deleting ASIC1a, which abolishes acidosis-induced $\left[\mathrm{Ca}^{2+}\right]_{\mathrm{i}}$ increase in spines (Zha et al., 2006), excluded the effect of acidosis on spine reduction. It will be of future interest to test whether acidosis-induced spine loss requires neural activity. Further, our data confirm the importance of ASIC1a maturation and trafficking in this process. Expressing N366Q, which increased maturation and dendritic targeting of ASIC1a, potentiated acidosis-induced spine loss at 
both pH 6 and 6.5. Conversely, expressing N393Q, which showed defective glycosylation and trafficking, had the opposite effect. One puzzling finding is that acidosis increased spine density of ASIC1a $^{-/-}$slice neurons or N393Q transfected neurons. These data suggest that acidosis also activates an ASIC1a-independent process, which leads to an increase in spine number. The exact mechanism for this effect will be of interest for future investigations.

In addition to spine numbers, spine geometry also plays critical roles in the integration and amplification of synaptic signals (Koch and Zador, 1993; Bloodgood and Sabatini, 2007). In particular, spine length controls the diffusion of signals or coupling between spine head and dendritic shaft (Majewska et al., 2000; Bloodgood and Sabatini, 2005; Noguchi et al., 2005). Here, we found that acidosis also regulates spine length in an ASICladependent manner. While further studies will be needed to directly assess the exact physiological significance of these changes, our results reveal for the first time a site-specific effect of acidosis on the dendritic region. Moreover, remodeling of dendritic spines, the sites for the majority of excitatory neurotransmission, correlates with changes in neural plasticity (Bhatt et al., 2009; Kasai et al., 2010). Thus, acidosis-induced spine remodeling further suggests that this process contributes to long-term changes in multiple neurological diseases.

\section{References}

Alvarez de la Rosa D, Krueger SR, Kolar A, Shao D, Fitzsimonds RM, Canessa CM (2003) Distribution, subcellular localization and ontogeny of ASIC1 in the mammalian central nervous system. J Physiol 546:77-87.

Benediktsson AM, Schachtele SJ, Green SH, Dailey ME (2005) Ballistic labeling and dynamic imaging of astrocytes in organotypic hippocampal slice cultures. J Neurosci Methods 141:41-53.

Bhatt DH, Zhang S, Gan WB (2009) Dendritic spine dynamics. Annu Rev Physiol 71:261-282.

Bloodgood BL, Sabatini BL (2005) Neuronal activity regulates diffusion across the neck of dendritic spines. Science 310:866-869.

Bloodgood BL, Sabatini BL (2007) $\mathrm{Ca}(2+)$ signaling in dendritic spines. Curr Opin Neurobiol 17:345-351.

Cai G, Salonikidis PS, Fei J, Schwarz W, Schülein R, Reutter W, Fan H (2005) The role of N-glycosylation in the stability, trafficking and GABA-uptake of GABA-transporter 1. Terminal N-glycans facilitate efficient GABAuptake activity of the GABA transporter. Febs J 272:1625-1638.

Canessa CM, Merillat AM, Rossier BC (1994) Membrane topology of the epithelial sodium channel in intact cells. Am J Physiol 267:C1682-C1690.

Chen S, Tran S, Sigler A, Murphy TH (2011) Automated and quantitative image analysis of ischemic dendritic blebbing using in vivo 2-photon microscopy data. J Neurosci Methods 195:222-231.

Cho JH, Askwith CC (2008) Presynaptic release probability is increased in hippocampal neurons from ASIC1 knockout mice. J Neurophysiol 99:426-441.

Chu XP, Wemmie JA, Wang WZ, Zhu XM, Saugstad JA, Price MP, Simon RP, Xiong ZG (2004) Subunit-dependent high-affinity zinc inhibition of acid-sensing ion channels. J Neurosci 24:8678-8689.

Chu XP, Close N, Saugstad JA, Xiong ZG (2006) ASIC1a-specific modulation of acid-sensing ion channels in mouse cortical neurons by redox reagents. J Neurosci 26:5329-5339.

Collier DM, Snyder PM (2009) Extracellular chloride regulates the epithelial sodium channel. J Biol Chem 284:29320-29325.

Collin C, Miyaguchi K, Segal M (1997) Dendritic spine density and LTP induction in cultured hippocampal slices. J Neurophysiol 77:1614-1623.

De Simoni A, Griesinger CB, Edwards FA (2003) Development of rat CA1 neurones in acute versus organotypic slices: role of experience in synaptic morphology and activity. J Physiol 550:135-147.

Duan B, Wang YZ, Yang T, Chu XP, Yu Y, Huang Y, Cao H, Hansen J, Simon RP, Zhu MX, Xiong ZG, Xu TL (2011) Extracellular spermine exacerbates ischemic neuronal injury through sensitization of ASICla channels to extracellular acidosis. J Neurosci 31:2101-2112.

Friese MA, Craner MJ, Etzensperger R, Vergo S, Wemmie JA, Welsh MJ, Vincent A, Fugger L (2007) Acid-sensing ion channel-1 contributes to axonal degeneration in autoimmune inflammation of the central nervous system. Nat Med 13:1483-1489.

Gao J, Duan B, Wang DG, Deng XH, Zhang GY, Xu L, Xu TL (2005) Coupling between NMDA receptor and acid-sensing ion channel contributes to ischemic neuronal death. Neuron 48:635-646.

Gonzales EB, Kawate T, Gouaux E (2009) Pore architecture and ion sites in acid-sensing ion channels and P2X receptors. Nature 460:599-604.

Hebert DN, Garman SC, Molinari M (2005) The glycan code of the endoplasmic reticulum: asparagine-linked carbohydrates as protein maturation and quality-control tags. Trends Cell Biol 15:364-370.

Helenius A, Aebi M (2004) Roles of N-linked glycans in the endoplasmic reticulum. Annu Rev Biochem 73:1019-1049.

Immke DC, McCleskey EW (2001) Lactate enhances the acid-sensing $\mathrm{Na}+$ channel on ischemia-sensing neurons. Nat Neurosci 4:869-870.

Jasti J, Furukawa H, Gonzales EB, Gouaux E (2007) Structure of acidsensing ion channel 1 at 1.9 A resolution and low pH. Nature 449:316-323.

Jiang Q, Li MH, Papasian CJ, Branigan D, Xiong ZG, Wang JQ, Chu XP (2009) Characterization of acid-sensing ion channels in medium spiny neurons of mouse striatum. Neuroscience 162:55-66.

Jiang Q, Papasian CJ, Wang JQ, Xiong ZG, Chu XP (2010) Inhibitory regulation of acid-sensing ion channel 3 by zinc. Neuroscience 169:574-583.

Jiang Q, Inoue K, Wu X, Papasian CJ, Wang JQ, Xiong ZG, Chu XP (2011) Cysteine 149 in the extracellular finger domain of acid-sensing ion channel $1 \mathrm{~b}$ subunit is critical for zinc-mediated inhibition. Neuroscience 193:89-99.

Jing L, Jiang YQ, Jiang Q, Wang B, Chu XP, Zha XM (2011) Interaction between the first transmembrane domain and the thumb of acid-sensing ion channel 1a is critical for its $\mathrm{N}$-glycosylation and trafficking. PLoS One 6:e26909.

Kadurin I, Golubovic A, Leisle L, Schindelin H, Gründer S (2008) Differential effects of $\mathrm{N}$-glycans on surface expression suggest structural differences between the acid-sensing ion channel (ASIC) 1a and ASIC1b. Biochem J 412:469-475.

Kasai H, Fukuda M, Watanabe S, Hayashi-Takagi A, Noguchi J (2010) Structural dynamics of dendritic spines in memory and cognition. Trends Neurosci 33:121-129.

Koch C, Zador A (1993) The function of dendritic spines: devices subserving biochemical rather than electrical compartmentalization. J Neurosci 13:413-422.

Li JG, Chen C, Liu-Chen LY (2007) N-glycosylation of the human kappa opioid receptor enhances its stability but slows its trafficking along the biosynthesis pathway. Biochemistry 46:10960-10970.

Majewska A, Brown E, Ross J, Yuste R (2000) Mechanisms of calcium decay kinetics in hippocampal spines: role of spine calcium pumps and calcium diffusion through the spine neck in biochemical compartmentalization. J Neurosci 20:1722-1734.

Noël J, Salinas M, Baron A, Diochot S, Deval E, Lingueglia E (2010) Current perspectives on acid-sensing ion channels: new advances and therapeutic implications. Expert Rev Clin Pharmacol 3:331-346.

Noguchi J, Matsuzaki M, Ellis-Davies GC, Kasai H (2005) Spine-neck geometry determines NMDA receptor-dependent $\mathrm{Ca} 2+$ signaling in dendrites. Neuron 46:609-622.

Oliva AA Jr, Lam TT, Swann JW (2002) Distally directed dendrotoxicity induced by kainic acid in hippocampal interneurons of green fluorescent protein-expressing transgenic mice. J Neurosci 22:8052-8062.

Prescher JA, Bertozzi CR (2006) Chemical technologies for probing glycans. Cell 126:851-854.

Quirk PL, Rao S, Roth BL, Siegel RE (2004) Three putative N-glycosylation sites within the murine 5-HT3A receptor sequence affect plasma membrane targeting, ligand binding, and calcium influx in heterologous mammalian cells. J Neurosci Res 77:498-506.

Rotin D, Kanelis V, Schild L (2001) Trafficking and cell surface stability of ENaC. Am J Physiol Renal Physiol 281:F391-399.

Schlote W, Betz E, Nguyen-Duong H (1975) Reversible apical swelling of dendrites in the cerebral cortex of cats during respiratory acidosis. Adv Neurol 12:483-495.

Sherwood TW, Askwith CC (2008) Endogenous arginine-phenylalanineamide-related peptides alter steady-state desensitization of ASIC1a. J Biol Chem 283:1818-1830.

Sherwood TW, Askwith CC (2009) Dynorphin opioid peptides enhance 
acid-sensing ion channel 1a activity and acidosis-induced neuronal death. J Neurosci 29:14371-14380.

Siesjö BK (1982) Lactic acidosis in the brain: occurrence, triggering mechanisms and pathophysiological importance. Ciba Found Symp 87:77-100.

Sluka KA, Winter OC, Wemmie JA (2009) Acid-sensing ion channels: a new target for pain and CNS diseases. Curr Opin Drug Discov Devel 12:693-704.

Vacca F, D'Ambrosi N, Nestola V, Amadio S, Giustizieri M, Cucchiaroni ML, Tozzi A, Velluz MC, Mercuri NB, Volonté C (2011) N-Glycans mutations rule oligomeric assembly and functional expression of $\mathrm{P} 2 \mathrm{X} 3$ receptor for extracellular ATP. Glycobiology 21:634-643.

Vukicevic M, Kellenberger S (2004) Modulatory effects of acid-sensing ion channels on action potential generation in hippocampal neurons. Am J Physiol Cell Physiol 287:C682-690.

Waldmann R, Champigny G, Bassilana F, Heurteaux C, Lazdunski M (1997) A proton-gated cation channel involved in acid-sensing. Nature 386: 173-177.

Wemmie JA, Chen J, Askwith CC, Hruska-Hageman AM, Price MP, Nolan BC, Yoder PG, Lamani E, Hoshi T, Freeman JH Jr, Welsh MJ (2002) The acid-activated ion channel ASIC contributes to synaptic plasticity, learning, and memory. Neuron 34:463-477.

Wemmie JA, Askwith CC, Lamani E, Cassell MD, Freeman JH Jr, Welsh MJ (2003) Acid-sensing ion channel 1 is localized in brain regions with high synaptic density and contributes to fear conditioning. J Neurosci 23:5496-5502.

Xiong ZG, Zhu XM, Chu XP, Minami M, Hey J, Wei WL, MacDonald JF, Wemmie JA, Price MP, Welsh MJ, Simon RP (2004) Neuroprotection in ischemia: blocking calcium-permeable acid-sensing ion channels. Cell 118:687-698.

Yermolaieva O, Leonard AS, Schnizler MK, Abboud FM, Welsh MJ (2004) Extracellular acidosis increases neuronal cell calcium by activating acidsensing ion channel 1a. Proc Natl Acad Sci U S A 101:6752-6757.

Zha XM, Green SH, Dailey ME (2005) Regulation of hippocampal synapse remodeling by epileptiform activity. Mol Cell Neurosci 29:494-506.

Zha XM, Wemmie JA, Green SH, Welsh MJ (2006) Acid-sensing ion channel la is a postsynaptic proton receptor that affects the density of dendritic spines. Proc Natl Acad Sci U S A 103:16556-16561.

Zha XM, Wang R, Collier DM, Snyder PM, Wemmie JA, Welsh MJ (2009a) Oxidant regulated inter-subunit disulfide bond formation between ASIC1a subunits. Proc Natl Acad Sci U S A 106:3573-3578.

Zha XM, Costa V, Harding AM, Reznikov L, Benson CJ, Welsh MJ (2009b) ASIC2 subunits target acid-sensing ion channels to the synapse via an association with PSD-95. J Neurosci 29:8438-8446.

Ziemann AE, Schnizler MK, Albert GW, Severson MA, Howard MA 3rd, Welsh MJ, Wemmie JA (2008) Seizure termination by acidosis depends on ASIC1a. Nat Neurosci 11:816-822. 